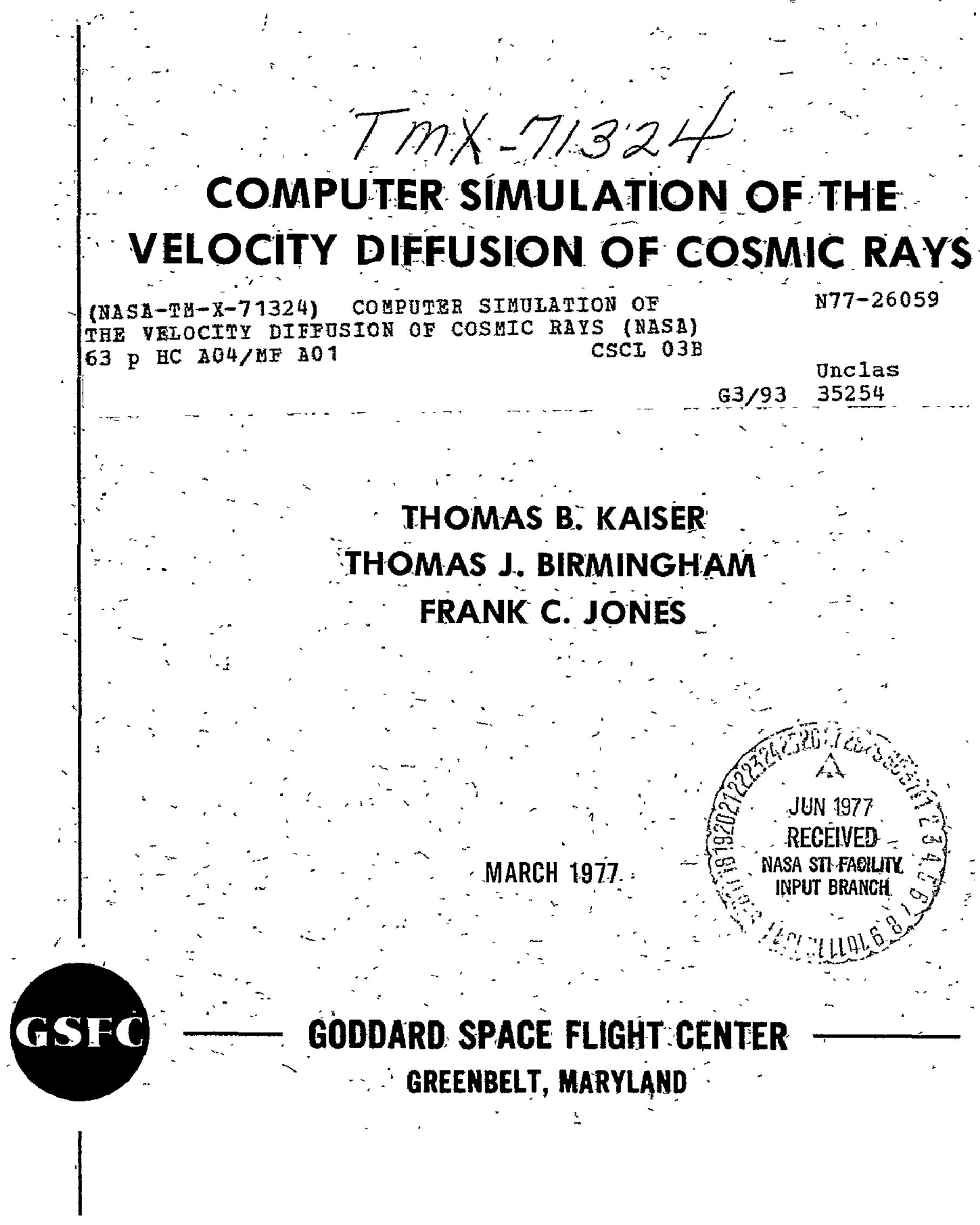




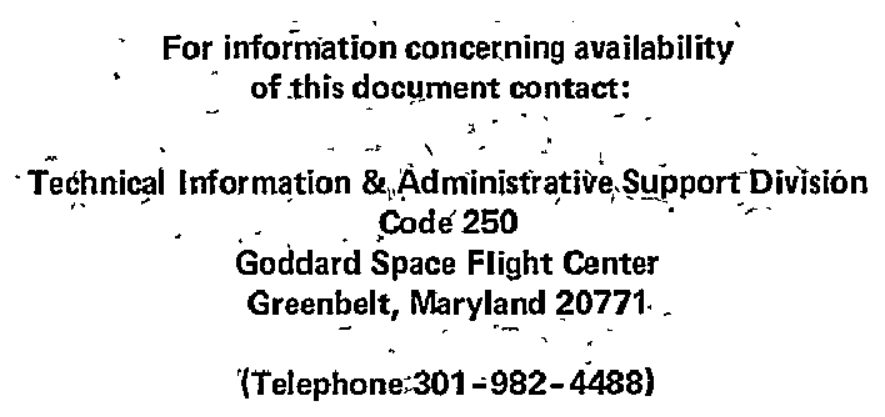

"This paper presents the views'of the author(s), and does not necessarily reflect the views of the Goddard Space Flight Center, or NASA.." . 


\section{COMPUTER SIMULATION OF THE VELOCITY DIFFUSION OF COSMIC RAYS}

Thomas B. Kaiser*

Thomas J. Birmingham

Frank C. Jones

March 1977

*NAS-NRC Resident Research Associate. Present Address:

Lawrence Livermore Laboratory, P.O. Box 808, Livermore, CA.

GODDARD SPACE FLIGHT CENTER

Greenbelt, Maryland 
COMPUTER SIMULATION OF THE VELOCITY DIFFUSION

\author{
OF COSMIC RAYS \\ Thomas B. Kaiser \\ Thomas J. Birmingham \\ Frank C. Jones
}

\begin{abstract}
Monte Carlo simulation experiments have been performed in order to study the velocity diffusion of charged particles in a static turbulent magnetic field. By following orbits of particles moving in a large ensemble of random magnetic field realizations with suitably chosen statistical properties, a pitch-angle diffusion coefficient is derived. Results are presented for a variety of particle rigidities and $\mathrm{rms}$ random field strengths and compared with the predictions of standard quasi-linear theory and the non-linear partially-averaged field theory described in a companion paper.
\end{abstract}


CONTENTS

$\underline{\text { Page }}$

ABSTRACT . . . . . . . . . . . . . . . . . . . . i . . . . . .

I. INTRODUCTION ...................... 1

II. THE MAGNETIC FIELD. . . . . . . . . . . . . . . 3

III. THE TIME DEPENDENT EXPERTMENT . . . . • . . . . . 5

IV. THE STEADY STATE EXPERIMENT: DESIGN AND INTERPRETATION . . . . . . . . . . . . . . . 9

a. Experimental Design . . . . . . . . . . . . . . . 9

b. Computer Implementation . . . . . . . . . . . . 10

c. Results of a Typical Experiment . . . . . . . . . . . 14

d. Determination of $D_{\mu \mu}(\mu, \infty)$. . - . . . . . . 15

e. Experimental Justification of Approximations . . . . . . . 16

1. Gyrotropy . . . . . . . . . . . . . . . . 17

2. Boundary Conditions . . . . . . . . . . . . 18

3. Adiabatic Approximation . . . . . . . . . . . 18

V. RESULTS . . . . . . . . . . . . . . . . . 20

APPENDIX A - The Particle Pusher . . . . . . . . . . . A-1

. T. THE ALGORITHM .................. A-1

II. PROPERTIES OF THE ALGORITHM . . . . . . . . . . A-4

à. Time Reversibility .. . . . . . . . . . . . . . A-4

b. Conservation of Energy . . . . . . . . . . . . . A-5

c. Accuracy . : . . . . . . . . . . . . . A-6

d. Computational Speed................... . A-8

APPENDIX B - Boundary Conditions for the Steady State Experiment ................ B-1

APPENDIX C - Error Analysis . . . . . . . . . . . . . C-1 


\section{COMPUTER SIMULATION OF THE VELOCITY DIFFUSION \\ OF COSMIC RAYS}

\section{INTRODUCTION}

It is now widely acknowledged that the velocity diffusion of charged particles induced by random fluctuations in a static magnetic field can be fully understood only in terms of a non-linear theory. ${ }^{1}$ The quasi-linear analysis of the process, which works well in most regions of phase space, breaks down in the case of particles whose pitch angles relative to the average magnetic field, $\theta$, approach $90^{\circ}$.

During the past few years, several non-linear theories have been proposed. The accompanying paper, ${ }^{2}$ hereafter referred to as Paper I, gives a detailed presentation of one such theory along with a list of references to several others. All of them, as well as the quasi-linear treatment, compute coefficients for diffusion in $\mu=\cos \theta$ which are mutually consistent for $|\mu| \rightarrow 1$. For $\mu \rightarrow 0$, however, they obtain results which vary widely as to their dependence on pitch angle, rigidity and random field strength.

Choice among the competing non-linear theories has been difficult because, on the one hand, none of them includes rigorous internal validity criteria and, on the other, no experimental observations with which to compare their divergent predictions are currently available.

In order to help remedy this situation we have performed computer simulation experiments for a variety of particle rigidities and $\mathrm{rms}$ random field 
strengths. In this way we have been able to study the diffusion process directly, independent of any theoretical assumptions. This has made it possible to clearly delineate the region of phase space throughout which quasi-linear theory breaks down and to derive the pitch angle diffusion coefficient as a function of $\mu$ for a range of experimental parameters.

We use the term "experiment" to describe the following procedure. A distribution of particles in phase space is allowed to evolve under a particular set of initial and boundary conditions in one realization chosen from a previously prepared statistical ensemble of stochastic magnetic fields. Exact particle orbits in the given field are followed, and information about the time evolution of the distribution is stored in the form of various reduced distribution functions and their moments. This process is then repeated for each of the several hundred magnetic field realizations comprising the ensemble, and, finally, the results are ensemble averaged.

Two qualitatively different sets of initial and boundary conditions were used. With one set the transient relaxation of an initially anisotropic distribution of particles was followed. This design was used primarily as a test of the basic simulation method and the computer codes used to implement it. Under the other set of conditions a steady state was established by uniform injection and absorption of particles. It was from this latter design that we derived our principal experimental results.

The magnetic field model and the method used to generate the ensemble are described in Section II. In Section III the time-dependent experiment is 
discussed. Section IV gives a detailed description of the design and interpretation of the steady-state experiments, and Section $V$ presents their results for a variety of parameter sets. The formulation and performance of the particle orbit integration algorithm, a derivation of the appropriate boundary conditions for the steady-state experiments, and an analysis of the uncertainty in the experimental diffusion coefficient are treated in appendices.

II. THE MAGNETIC FIELD

For reasons given in Paper I, each realization of the magnetic field was taken to be of the form

$$
\underset{\sim}{\mathrm{B}}(\mathrm{r})=\hat{\mathrm{e}}_{\mathrm{x}} \delta \mathrm{B}(\mathrm{z})+\hat{\mathrm{e}}_{\mathrm{z}}\langle\mathrm{B}\rangle
$$

with $\delta \mathrm{B}(\mathrm{z})$ a homogeneous Gaussian process ${ }^{3}$ of mean zero and two-point correlation function

$$
\begin{aligned}
\mathrm{C}_{\mathrm{B}}\left(\mathrm{z}^{\prime}-\mathrm{z}\right) & =\left\langle\delta \mathrm{B}(\mathrm{z}) \delta \mathrm{B}\left(\mathrm{z}^{\prime}\right)\right\rangle /\left\langle\delta \mathrm{B}^{2}\right\rangle \\
= & \exp \left[-\frac{\left|\mathrm{z}^{\prime}-\mathrm{z}\right|}{\mathrm{z}_{\mathrm{c}}}\right]
\end{aligned}
$$

which corresponds to the power spectrum

$$
\begin{aligned}
P(k)= & \int_{-\infty}^{\infty} \mathrm{d} \zeta \mathrm{e}^{-\mathrm{i} k \zeta} \mathrm{C}_{\mathrm{B}}(\zeta), \\
= & \frac{2 \mathrm{z}_{\mathrm{c}}}{1+\mathrm{k}^{2} \mathrm{z}_{\mathrm{c}}^{2}}
\end{aligned}
$$

The choice of an exponential correlation function ensures that $\delta \mathrm{B}(\mathrm{z})$ is a Markovian process, i. e., one that is completely specified by its two-point probability density. ${ }^{4}$ 
A realization of such a process can be generated on a grid of spacing $h$ by the following procedure. .

1. Choose $\delta \mathrm{B}(0)$ from a Gaussian distribution with mean zero and variance $\left\langle\delta \mathrm{B}^{2}\right\rangle$

2. Let $\mathrm{P}\left[\delta \mathrm{B}\left(\mathrm{z}^{\prime}\right) \mid \delta \mathrm{B}(\mathrm{z})\right]$ be the conditional probability density for $\delta \mathrm{B}\left(\mathrm{z}^{\prime}\right)$, given that $\delta \mathrm{B}$ has the value $\delta \mathrm{B}(\mathrm{z})$ at $\mathrm{z}$. It is straightforward to show that $\mathrm{P}\left[\delta \mathrm{B}\left(\mathrm{z}^{\prime}\right) \mid \delta \mathrm{B}(\mathrm{z})\right]$ is Gaussian with mean $\delta \mathrm{B}(\mathrm{z}) \mathrm{C}_{\mathrm{B}}\left(\mathrm{z}^{\prime}-\mathrm{z}\right)$ and variance $\left\langle\delta B^{2}\right\rangle\left[1-C_{B}\left(z^{\prime}-z\right)^{2}\right]$. Choose $\delta B(h)$ from a distribution with density $\mathbf{P}[\delta \mathrm{B}(\mathrm{h}) \mid \delta \mathrm{B}(0)]$.

3. Repeat step (2) for succeeding grid points, each time selecting $\delta \mathrm{B}(\mathrm{nh})$ from a distribution with density $\mathrm{P}[\delta \mathrm{B}(\mathrm{nh}) \mid \delta \mathrm{B}(\mathrm{nh}-\mathrm{h})]$. Since $\delta \mathrm{B}(\mathrm{z})$ is a Markovian process, the distribution of $\delta \mathrm{B}(\mathrm{nh})$ depends only on $\delta \mathrm{B}(\mathrm{nh}-\mathrm{h})$ and not on $\delta \mathrm{B}(\mathrm{nh}-\mathrm{mh}), 2 \leqslant \mathrm{~m} \leqslant \mathrm{n}$.

This method was used to generate an ensemble of 800 realizations of the random component of the magnetic field. Each realization was defined on a grid of length $200 \mathrm{z}_{\mathrm{c}}$ and spacing $\mathrm{h}=\mathrm{z}_{\mathrm{c}} / 64$ and had $\left\langle\delta \mathrm{B}^{2}\right\rangle=1$. Between grid points values of the field were defined by linear interpolation. The ensemble was stored on magnetic tape and used repeatedly. During the performance of an experiment the realizations were read sequentially and the field values scaled to give the desired rms random field strength. A segment of a typical realization is shown in Figure 1.

If the empirical correlation function of $\delta \mathrm{B}(\mathrm{z})$ over a finite ensemble of $\mathrm{R}$ realizations is defined 


$$
\begin{gathered}
\Gamma_{\mathrm{B}}^{\mathrm{R}}(\mathrm{z}) \equiv \frac{1}{\left\langle\delta \mathrm{B}^{2}\right\rangle(\mathrm{R}-1)} \sum_{\mathrm{n}=1}^{\mathrm{R}}\left\{\left[\delta \mathrm{B}^{(\mathrm{n})}(\mathrm{o})-\frac{1}{\mathrm{R}} \sum_{\mathrm{m}=1}^{\mathrm{R}} \delta \mathrm{B}^{(\mathrm{m})}(\mathrm{o})\right]\right. \\
\left.\cdot\left[\delta \mathrm{B}^{(\mathrm{n})}(\mathrm{z})-\frac{1}{\mathrm{R}} \sum_{\ell=1}^{\mathrm{R}} \delta \mathrm{B}^{(\mathrm{l})}(\mathrm{z})\right]\right\},
\end{gathered}
$$

where the superscripts $n, m$, $l$ on the right side index the finite ensemble, then it is straightforward to show. that the mean and standard deviation of $\Gamma_{B}^{R}(z)$ are

$$
\begin{gathered}
\left\langle\Gamma_{\mathrm{B}}^{\mathrm{R}}(\mathrm{z})\right\rangle=\mathrm{C}_{\mathrm{B}}(\mathrm{z}), \\
\left\langle\left[\Gamma_{\mathrm{B}}^{\mathrm{R}}(\mathrm{z})-\left\langle\Gamma_{\mathrm{B}}^{\mathrm{R}}(\mathrm{z})\right\rangle\right]^{2}\right\rangle^{1 / 2} \cong\left[\frac{1+\mathrm{C}_{\mathrm{B}}(\mathrm{z})^{2}}{\mathrm{R}}\right]^{1 / 2}
\end{gathered}
$$

In the above, the angular brackets indicate averages over the infinite Gibbsian ensemble.

The right side of Equation (4) for the full 800-realization ensemble is shown in Figure 2 compared with the theoretical expression, Equation (2). Onestandard-deviation limits, as computed from Equation (6) are also shown.

\section{THE TIME DEPENDENT EXPERIMENT}

In order to check the validity of our simulation method and the computer codes used to implement it, an experiment was designed to monitor the evolution of a distribution of particles in a region of phase space where quasi-linear theory is valid and the diffusion process. well understood.

In this experiment we followed the mean and variance of the space and gyrophase averaged distribution function, $f_{o}(\mu, t)$, as it evolved from the initial condition 


$$
f_{0}(\mu, 0)=\delta\left(\mu-\mu_{0}\right)
$$

This was accomplished by integrating the trajectory of a single particle in each of $R$ realizations of the ensemble of random magnetic fields. Each particle had the common starting point $\mu(t=0)=\mu_{0}$ and a velocity phase angle chosen randomly from a uniform distribution on $[0,2 \pi]$, and was followed for the same length of time. The mean and variance were computed as

$$
\begin{gathered}
m(t)=\langle\mu(t)\rangle_{R}=\frac{1}{R} \sum_{n=1}^{R} \mu^{(n)}(t) \\
\sigma^{2}(t)=\left\langle[\mu(t)-\langle\mu(t)\rangle]^{2}\right\rangle_{R}=\frac{1}{R-1} \sum_{n=1}^{R}[\mu(n)(t)-\langle\mu(t)\rangle]^{2}
\end{gathered}
$$

where the superscript (n) indexes realizations over the ensemble.

The behavior of $m(t)$ and $\sigma^{2}(t)$ predicted by quasi-linear theory can be obtained from the kinetic equation for $f_{0} 5$

$$
\begin{gathered}
\frac{\partial \mathrm{f}_{\mathrm{o}}}{\partial \mathrm{t}}=\frac{\partial}{\partial \mu}\left[\mathrm{D}_{\mu \mu}^{\mathrm{QL}}(\mu, \mathrm{t}) \frac{\partial \mathrm{f}_{\mathrm{o}}}{\partial \mu}\right] \\
D_{\mu \mu}^{\mathrm{QL}}( \pm 1, \mathrm{t}) \frac{\partial \mathrm{f}_{\mathrm{o}}}{\partial \mu}=0
\end{gathered}
$$

where the quasi-linear diffusion coefficient is ${ }^{6}$

$$
\begin{gathered}
\mathrm{D}_{\mu \mu}^{\mathrm{QL}}(\mu, \mathrm{t})=\mathrm{D}^{\mathrm{QL}}(\mu, \infty)[1+\mathrm{J}(\mu, \mathrm{t})] \\
\mathrm{D}^{\mathrm{QL}}(\mu, \infty)=\frac{\mathrm{z}_{\mathrm{c}}}{\mathrm{v}} \frac{\left\langle\delta \omega^{2}\right\rangle}{2} \frac{|\mu|\left(1-\mu^{2}\right)}{\mu^{2}+\mathrm{z}_{\mathrm{c}}^{2} \omega_{\mathrm{o}}^{2} / \mathrm{v}^{2}} \\
\mathrm{~J}(\mu, \mathrm{t})=\mathrm{e}^{-|\mu| \mathrm{vt} / \mathrm{z}_{\mathrm{c}}}\left[\cos \omega_{\mathrm{o}} \mathrm{t}-\frac{\mathrm{z}_{\mathrm{c}} \omega_{\mathrm{o}}}{|\mu| \mathrm{v}} \sin \omega_{\mathrm{o}} \mathrm{t}\right]
\end{gathered}
$$


with

$$
\begin{gathered}
\left\langle\delta \omega^{2}\right\rangle=\frac{\mathrm{q}^{2}\left\langle\delta \mathrm{B}^{2}\right\rangle}{\gamma^{2} \mathrm{~m}^{2} \mathrm{c}^{2}}, \\
\omega_{\circ}=\frac{\mathrm{q}\langle\mathrm{B}\rangle}{\gamma \mathrm{mc}} .
\end{gathered}
$$

The transient component, $\mathrm{J}(\mu, \mathrm{t})$, is included for completeness. Its effect damps out on the correlation time scale, $\mathrm{t}_{\mathrm{c}}^{\mathrm{QL}}=\mathrm{z}_{\mathrm{c}}(\mathrm{v}|\mu|)^{-1}$, which in the quasilinear regime is comparable to the transit time, $z_{c} / v$. The contribution of $J(\mu, t)$ to $m(t)$ was unobservably small in the time-dependent experiment, and while observable, its small contribution to $\sigma^{2}(t)$ damped in a few transit times. Consequently, in calculating $m(t)$ and $\sigma^{2}(t)$ from Equations $(10)-(12), J(\mu, t)$ will be ignored.

Integration of Equation (10) forward in time yields

$$
\mathrm{f}_{\mathrm{o}}(\mu, \mathrm{t})=\delta\left(\mu-\mu_{0}\right)+\int_{0}^{\mathrm{t}} \mathrm{dt} \mathrm{t}^{\prime} \frac{\partial}{\partial \mu}\left[\mathrm{D}_{\mu \mu}^{\mathrm{QL}}(\mu, \infty) \frac{\partial \mathrm{f}_{\mathrm{o}}\left(\mu, \mathrm{t}^{\prime}\right)}{\partial \mu}\right]
$$

The mean value of $\mu$ is then

$$
\mathrm{m}(\mathrm{t})=\int_{-1}^{+1} \mathrm{~d} \mu \mu \mathrm{f}_{\mathrm{o}}(\mu, \mathrm{t})=\mu_{\mathrm{o}}+\int_{0}^{\mathrm{t}} \mathrm{dt^{ \prime }} \int_{-1}^{+1} \mathrm{~d} \mu \mu \frac{\partial}{\partial \mu}\left[\mathrm{D}_{\mu \mu}^{\mathrm{QL}}(\mu, \infty) \frac{\partial \mathrm{f}_{\mathrm{o}}\left(\mu, \mathrm{t}^{\prime}\right)}{\partial \mu}\right]
$$

which may be integrated twice by parts using the boundary conditions, Equation (11), to give

$$
\mathrm{m}(\mathrm{t})=\mu_{\mathrm{o}}+\int_{0}^{\mathrm{t}} \mathrm{dt^{ \prime }} \int_{-1}^{+1} \mathrm{~d} \mu \frac{\partial}{\partial \mu}\left[\mathrm{D}_{\mu \mu}^{\mathrm{QL}}(\mu, \infty)\right] \mathrm{f}_{\mathrm{o}}\left(\mu, \mathrm{t}^{\prime}\right)
$$

Furthermore, in the region of $\mu$-space covered by this experiment, the variation of $\mathrm{D}_{\mu \mu}^{\mathrm{QL}}(\mu, \infty)$ is very nearly linear 


$$
\mathrm{D}_{\mu \mu}^{\mathrm{QL}}(\mu, \infty) \cong \mathrm{D}_{\mathrm{o}}^{\mathrm{QL}}+\mathrm{D}_{1}^{\mathrm{QL}}\left(\mu-\mu_{\mathrm{o}}\right),
$$

Upon using this form plus the normalization

$$
\int_{-i}^{+1} d \mu f_{0}\left(\mu, t^{\prime}\right)=1
$$

we obtain

$$
m(t)=\mu_{o}+D_{1}^{Q \mathbf{L}} t
$$

The variance of $f_{0}$,

$$
\sigma^{2}(\mathrm{t}) \equiv \int_{-1}^{1} \mathrm{~d} \mu[\mu-\mathrm{m}(\mathrm{t})]^{2} \mathrm{f}_{\mathrm{o}}(\mu, \mathrm{t})
$$

can be derived in a similar fashion by computing the second moment of Equation (15). The result is

$$
\sigma^{2}(t)=2 D_{0}^{Q L} t+\left(D_{1}^{Q L} t\right)^{2}
$$

Data for the case $\mathrm{z}_{\mathrm{c}} \omega_{0} / \mathrm{v}=1, \mathrm{z}_{\mathrm{c}}\left\langle\delta \omega^{2}\right\rangle^{1 / 2} / \mathrm{v}=10^{-3 / 2}, \mathrm{R}=200, \mu_{\mathrm{o}}=(3 / 4)^{1 / 2}$ are given in Figures 3 and 4 with Equations (19) and (21) plotted for comparison. Note especially that the transient component of $\mathrm{D}_{\mu \mu}^{\mathrm{QL}}$ has negligible effect on $\mathrm{m}(t)$, and its effect on $\sigma^{2}(t)$, which is responsible for the initial depression below the value given by Equation (21), damps completely in a few transit times. The excellent agreement between the behavior predicted by quasi-linear theory and that actually observed in the computer simulation strongly indicates that the simulation code accurately depicts the physical phenomena. 
IV. THE STEADY STATE EXPERIMENT: DESIGN AND INTERPRETATION

a. Experimental Design

In order to study the diffusion process in regions of phase space where quasi-linear theory breaks down, experiments of the type described in Section III were found to be inadequate. Transient effects damp so slowly that by the time they are negligible the initial distribution function has spread to the point where Equation (18) is badly violated. In other words, the correlation time becomes comparable to the relaxation time of the initial distribution. An illustration of this behavior is given in Figure 5, in which $\sigma^{2}(\mathrm{t})$ as computed from Equations (8) and (9) is plotted for the ease $z_{c} \omega_{0} / v=1, z_{c}\left\langle\delta \omega^{2}\right\rangle^{1 / 2} / v=10^{-3 / 2}$, $R=200$ and $\mu_{0}=0$. The initial transient oscillations are seen to persist for more than 50 transit times and clearly are not negligible. By the time they have apparently damped away, the distribution has relaxed to a point where its evolution is no longer characterized by the value of the diffusion coefficient at $\mu=\mu_{0}$.

An obvious way to circumvent this difficulty is to design an experiment in which the relaxation time is very long, in fact infinite, i. e., one in which the particle distribution has evolved to a steady state.

Of course, the only steady-state solution of a diffusion equation like Equation (10) with boundary conditions Equation (11) is the uninteresting isotropic distribution, $f_{o}=$ constant. This situation would be altered, however, if a source of particles and absorbing boundaries were introduced. Then, when the influx of particles from the source was balanced by the escape of particles through the 
boundaries, a steady state would be attained. Physically, such a situation would be similar to the injection of particles into a magnetic mirror field and their subsequent escape by diffusion into the loss cones.

\section{b. Computer Implementation}

The steady state experiment was executed in the following way. A total of $\mathrm{N}$ particles with independent and uniformly distributed velocity phase angles were launched at $t=0$ at the point $\underset{\sim}{\underset{\sim}{r}}=\underset{\sim}{\underline{S}}$ with $\mu=\mu_{\mathrm{S}}$ in each of $\mathrm{R}$ realizations of the magnetic field. Typically $\mathrm{N}$ was $10-20$ and $\mathrm{R}$ was 800 . Absorbing boundaries were located at $\mu=\mu_{\mathrm{L}}<\mu_{\mathrm{S}}$ and $\mu=\mu_{\mathrm{R}}>\mu_{\mathrm{S}}$.

The trajectory of each particle was followed until it left the region $\mu_{\mathrm{L}} \leqslant \mu \leqslant \mu_{\mathrm{R}}$, at which time the particle was annihilated. Snapshots of the evolving particle distribution function in each realization were taken at regular time intervals $\Delta t$ for a total time $t_{F}$, long enough for all particles to be absorbed. The $R$ collections, of $K=t_{F} / \Delta t$ snapshots each, thus generated were then averaged over the $R$ realizations of the magnetic field to give an ensemble averaged collection of snapshots, $\langle G\rangle_{R}\left(\mu, t_{k} \mid \mu_{S}, 0\right), t_{k}=k \Delta t, k=0,1, \ldots, K$.

We show now that

$$
\begin{aligned}
& \langle f\rangle_{\mathrm{R}}=\sum_{k=1}^{\mathrm{K}}\langle\mathrm{G}\rangle_{\mathrm{R}}\left(\mu, \mathrm{t}_{\mathrm{k}} \mid \mu_{\mathrm{S}}, 0\right) \\
& \cong \frac{1}{\Delta \mathrm{t}} \int_{0}^{\infty} \mathrm{d} \tau\langle\mathrm{G}\rangle_{\mathrm{R}}\left(\mu, \tau \mid \mu_{\mathrm{S}}, 0\right)
\end{aligned}
$$

satisfies approximately the steady-state diffusion equation 


$$
\frac{\mathrm{d}}{\mathrm{d} \mu}\left[\mathrm{D}_{\mu \mu}(\mu, \infty) \frac{\mathrm{d}\langle\mathrm{f}\rangle_{\mathrm{R}}}{\mathrm{d} \mu}\right]=-\frac{\mathrm{N}}{\Delta \mathrm{t}} \delta\left(\mu-\mu_{\mathrm{S}}\right)
$$

and equivalently that $\langle f\rangle_{R}$ is the steady-state one particle distribution function averaged over the finite ensemble.

To justify this assertion and approach, consider $\langle G\rangle_{R}\left(\mu, t\left[\mu_{S}, t^{\prime}\right)\right.$, the ensemble averaged Green's Function corresponding to an injection source of strength $N$ located at $\mu=\mu_{\mathrm{S}}$ at time $t^{\prime} .\langle\mathrm{G}\rangle_{\mathrm{R}}$ satisfies the equation

$$
\frac{\partial\langle G\rangle_{R}}{\partial t}=\frac{\partial}{\partial \mu}\left[D_{\mu \mu}\left(\mu, t-t^{\prime}\right) \frac{\partial\langle G\rangle_{R}}{\partial \mu}\right]+N \delta\left(\mu-\mu_{S}\right) \delta\left(t-t^{\prime}\right)
$$

the initial condition $\langle G\rangle_{R}\left(\mu, t \mid \mu_{S}, t^{\prime}\right)=0$ for $t<t^{\prime}$ and the boundary conditions, derived in Appendix B,

$$
\left.\left[\mathrm{D}_{\mu \mu}^{(\mu, \infty)} \frac{\partial\langle\mathrm{G}\rangle_{\mathrm{R}}}{\partial \mu} \pm\langle\alpha\rangle\langle\mathrm{G}\rangle_{\mathrm{R}}\right]\right|_{\mu=\mu_{\mathrm{R}}, \mu_{\mathrm{L}}}=0
$$

where the upper and lower signs apply at the absorbing boundaries at $\mu_{\mathrm{R}}$ and $\mu_{\mathrm{L}}$ respectively and $\langle\alpha\rangle$ is a $\mu$-dependent quantity given in the Appendix.' The "turn-on" time of the diffusion coefficient $D_{\mu \mu}$ is taken to be the injection time $t^{\prime}$, since fluctuations, $\delta G$, from realization to realization in the ensemble commence growth from zero at this time (cf. Paper I).

Note that since time enters Equation (24) only in the form $t-t^{\prime},\langle G\rangle_{R}$ is time translationally invariant

$$
\langle G\rangle_{R}\left(\mu, t \mid \mu_{S}, t^{\prime}\right) \equiv\langle G\rangle_{R}\left(\mu, t-t^{\prime} \mid \mu_{S}, 0\right)
$$


Consider then the quantity

$$
\int_{0}^{\mathrm{t}^{+}} \mathrm{d} \tau\langle\mathrm{G}\rangle_{\mathrm{R}}\left(\mu, \mathrm{t} \mid \mu_{\mathrm{S}}, \tau\right) \equiv \int_{0^{-}}^{\mathrm{t}} \mathrm{d} \tau\langle\mathrm{G}\rangle_{\mathrm{R}}\left(\mu, \tau \mid \mu_{\mathrm{S}}, 0\right)
$$

where, as in the usual Green's Function prescription, ${ }^{7}$ the integration extends to $t^{+}=t+\epsilon$. The relationship between $\langle f\rangle_{R}$ compiled in the computer experiment and the solution to Equation (24) is quite obvious

$$
\langle f\rangle_{R}=\lim _{t \rightarrow \infty}\langle F(t)\rangle_{R}=\lim _{t \rightarrow \infty} \frac{1}{\Delta t} \int_{0}^{t^{+}} d \tau\langle G\rangle_{R}\left(\mu, t ! \mu_{S}, \tau\right)
$$

To verify Equation (23), note that differentiation of $\langle F(t)\rangle_{R}$ with respect to time and use of Equation (24) and its initial condition result in

$$
\begin{gathered}
\frac{\partial\langle\mathrm{F}\rangle_{\mathrm{R}}}{\partial \mathrm{t}}=\frac{1}{\Delta \mathrm{t}} \int_{0}^{\mathrm{t}^{+}} \mathrm{d} \tau \frac{\partial\langle\mathrm{G}\rangle_{\mathrm{R}}\left(\mu, \mathrm{t} \mid \mu_{\mathrm{S}}, \tau\right)}{\partial \mathrm{t}} \\
=\frac{1}{\Delta \mathrm{t}} \int_{0}^{\mathrm{t}} \mathrm{d} \tau \frac{\partial}{\partial \mu}\left[\mathrm{D}_{\mu \mu}(\mu, \tau) \frac{\partial\langle\mathrm{G}\rangle_{\mathrm{R}}\left(\mu, \tau \mid \mu_{\mathrm{S}}, 0\right)}{\partial \mu}\right]+\frac{\mathrm{N}}{\Delta \mathrm{t}} \delta\left(\mu-\mu_{\mathrm{S}}\right)
\end{gathered}
$$

So long as $D_{\mu \mu}(\mu, t)$ is asymptotically positive, $i . e$. , that a time $t_{A}$ exists such that $D_{\mu \mu}(\mu, t) \geqslant \dot{0}$ if $t>t_{A}$, then an $H$-theorem for $\langle G\rangle_{R}$ is easily derived from Equations (24) and (25) by multiplying Equation (24) by $\langle G\rangle_{R}$ and integrating over $\mu$. Consequently $\langle\mathrm{G}\rangle_{\mathrm{R}}\left(\mu, \tau \mid \mu_{\mathrm{S}}, 0\right) \rightarrow 0$ as $\gamma \rightarrow \infty$ so that $\langle\mathrm{F}\rangle_{\mathrm{R}}$ approaches a steady state. Thus, asymptotically in time, Equation (26) reduces to

$$
\frac{\partial}{\partial \mu}\left[\int_{0}^{\infty} \mathrm{d} \tau \mathrm{D}_{\mu \mu}(\mu, \tau) \frac{\partial\langle\mathrm{G}\rangle_{\mathrm{R}}\left(\mu, \tau \mid \mu_{\mathrm{S}}, 0\right)}{\partial \mu}\right]=-\mathrm{N} \delta\left(\mu-\mu_{\mathrm{S}}\right)
$$

The diffusion coefficient $\mathrm{D} \mu \mu(\mu, \tau)$ saturates, i. e. , approaches the asymptotic value $D_{\mu \mu}(\mu, \infty)$, in a time of the order of the correlation time $\tau_{c} \cong z_{c} /(|\mu| v)$ 
of the fluctuating magnetic field (cf. Paper I). There is also a finite transport time of particles from the source point $\mu_{\mathrm{S}}$ to the point $\mu$. Roughly speaking $\tau_{\mathrm{tr}}^{1 / 2} \sim \frac{\left|\mu-\mu_{\mathrm{S}}\right|}{\left|\mathrm{D}_{\mu \mu}(\mu)\right|^{1 / 2}}$, so that $\langle\mathrm{G}\rangle_{\mathrm{R}} \cong 0$. for $\tau<\tau_{\mathrm{tr}}(\mu)$ and this region of small $\tau$ does not contribute to the integration in Equation (27).

Provided that

$$
\tau_{\mathrm{c}}(\mu) \leqslant \tau_{\mathrm{tr}}(\mu)
$$

we may then write

$$
\begin{gathered}
\frac{1}{\Delta t} \frac{\mathrm{d}}{\mathrm{d} \mu}\left[\mathrm{D}_{\mu \mu}(\mu, \infty) \frac{\mathrm{d}}{\mathrm{d} \mu} \int_{0}^{\infty} \mathrm{d} \tau\langle\mathrm{G}\rangle_{\mathrm{R}}\left(\mu, \tau \mid \mu_{\mathrm{S}}, 0\right)\right] \\
\quad=\frac{\mathrm{d}}{\mathrm{d} \mu}\left[\mathrm{D}_{\mu \mu}(\mu, \infty) \frac{\mathrm{d}}{\mathrm{d} \mu}\langle\mathrm{f}\rangle_{\mathrm{R}}\right]=-\frac{\mathrm{N}}{\Delta \mathrm{t}} \delta\left(\mu-\mu_{\mathrm{S}}\right)
\end{gathered}
$$

Note that in the immediate vicinity of the source Equation (28) is violated and hence Equation (29) is invalid. This violation is manifest, as we shall see, in the computer experiment. In our analysis of the computer results we shall quantify the region of validity in $\left(\mu-\mu_{\mathrm{S}}\right)$ of the approximate form Equation (29).

Note from Equation (27) that the particle fluxes

$$
\int_{0}^{\infty} \mathrm{d} \tau \mathrm{D}_{\mu \mu}(\mu, \tau) \frac{\partial\langle\mathrm{G}\rangle_{\mathrm{R}}\left(\mu, \tau \mid \mu_{\mathrm{S}}, 0\right)}{\partial \mu}
$$

to the left and right of the source are constant, independent of $\mu$. Thus in the region where Equation (29) is valid

$$
\mathrm{D}_{\mu \mu}(\mu, \infty) \frac{\mathrm{d}\langle\mathrm{f}\rangle_{\mathrm{R}}}{\mathrm{d} \mu}= \begin{cases}-\mathrm{J}_{\mathrm{L}} \cdot & \mu_{\mathrm{L}} \leqslant \mu<\mu_{\mathrm{S}} \\ -\mathrm{J}_{\mathrm{L}}-\frac{\mathrm{N}}{\Delta \mathrm{t}}=-\mathrm{J}_{\mathrm{R}} & \mu_{\mathrm{S}}<\mu \leqslant \mu_{\mathrm{R}}\end{cases}
$$

where $J_{L}$ and $J_{R}$ are the constant values of the left and right fluxes. 
In the computer experiment $\frac{\mathrm{d}\langle\mathrm{f}\rangle_{\mathrm{R}}}{\mathrm{d} \mu}, \mathrm{J}_{\mathrm{L}}$, and $\mathrm{J}_{\mathrm{R}}$ are directly and separately measured so that $D_{\mu \mu}(\mu, \infty)$ can be determined readily from Equation (30) independent of any theoretical assumptions save that Equations (24), (25), and (28) are valid.

c. Results of a Typical Experiment

In practice, $\langle f\rangle_{\mathrm{R}}(\mu)$ was obtained in the form of a histogram of bin width, $\Delta \mu$, typically $\cong 10^{-2}$. The result for the case $\mathrm{z}_{\mathrm{c}} \omega_{\mathrm{o}} / \mathrm{v}=1,\left\langle\delta \omega^{2}\right\rangle^{1 / 2} / \omega_{\mathrm{o}}=0.1$, $\mu_{\mathrm{L}}=-0.65, \mu_{\mathrm{S}}=0.405, \mu_{\mathrm{R}}=0.65, \Delta \mu=0.01,\left(\mathrm{v} / \mathrm{z}_{\mathrm{c}}\right) \Delta \mathrm{t}=0.03125, \mathrm{~N}=8$, $\mathbf{R}=800$, which was typical of the parameter regime throughout which simulations were performed, is given in Figure 6.

Before proceeding to a more detailed analysis of experimental results, we briefly discuss various gross features of the steady state distribution evident in Figure 6. Perhaps the most obvious characteristic of the distribution is the . presence of particles on the opposite side of $\mu=0$ from the source. The apparent ease with which particles scatter through $\theta=90^{\circ}$ is in direct contradiction to the prediction of quasi-linear theory, which yields a vanishing diffusion coefficient at $\mu=0$.

Also evident is a flattening of the distribution function just to the left of the source. Although the diffusion coefficient and, therefore, according to Equation (30), $\mathrm{d}\langle\mathrm{f}\rangle_{\mathrm{R}} / \mathrm{d} \mu$, are expected to be even functions of $\mu$, such is clearly not the case. We ascribe the lack of symmetry to the breakdown of the adiabatic approximation near the source and estimate the extent of the effect in Section IV-e. 
Finally, we call attention to the fluctuations in the number of particles occupying each histogram bin, the standard deviations of which comprised one of the diagnostics available in the experiments. It was observed that the magnitudes of these fluctuations, relative to the mean bin occupation numbers, were of order $R^{-1 / 2} \cong 0.04$, as would be expected on the basis of simple statistical arguments. This 'scaling gives an accurate measure of the' cost entailed in improving the statistical accuracy of the experiments.

\section{d. Determination of $\mathrm{D}_{\mu \mu}(\mu, \infty)$}

The diffusion coefficient, $\mathrm{D}_{\mu \mu}(\mu, \infty)$, was obtained from Equation (30), using the observed particle fluxes and the measured slope of the histogram representation of $\langle f\rangle_{R}(\mu)$.

The particle fluxes were determined by counting the number of particles that escaped through each boundary. Thus,

$$
\begin{gathered}
J_{L}=-\frac{N_{L}}{\Delta t}, \\
J_{R}=\frac{N_{R}}{\Delta t},
\end{gathered}
$$

where $N_{L}$ and $N_{R}$ are the ensemble averaged number of particles that escaped at $\mu=\mu_{L}$ and $\mu=\mu_{R}$, respectively.

The slope at a point, $\dot{\mu}$, was obtained from a linear least squares fit to the histogram points symmetrically bracketing $\mu$. Typically, ten points were used, the actual number being determined by requiring the statistical uncertainty in 
$\mathrm{D}_{\mu \mu}$ to be $\sim 10 \%$. A detailed analysis of the uncertainty in $\mathrm{D}_{\mu \mu}$ is given in Appendịx C.

The diffusion coefficient computed in this way from the data in Figure 6 is given in Figure 7. Also shown are the quasi-linear expression, Equation (13), and the diffusion coefficient derived in the partially averaged field theory described in Paper I.

Several details of the $\mu$-dependence of the experimental diffusion coefficient apparent in Figure 7 proved to be generally characteristic of $\mathrm{D}_{\mu \mu}(\mu, \infty)$ throughout the parameter regime studied, and we call attention to them here.

First of all, to within statistical uncertainty, $D_{\mu \mu}(\mu, \infty)$ is an even function of $\mu$. This fundamental property, predicted by all theories, is of interest chiefly as a check on the simulation scheme.

Furthermore, there is a significant peak in $\mathrm{D}_{\mu \mu}(\mu, \infty)$ at $\mu=0$. The en-. hanced scattering represented by this peak is predicted by the partially averaged field theory, ${ }^{2}$ which attributes the effect to particle mirroring.

Finally, the pitch angle regime throughout which quasi-linear theory fails is roughly characterized by $|\mu| \lesssim\left\langle\delta \mathrm{B}^{2}\right\rangle^{3 / 2} /\langle\mathrm{B}\rangle$. A theoretical explanation for this result is given in Paper $\mathrm{I}$.

e. Experimental Justification of Approximations

There were several approximations necessary to the derivation of the fundamental equations in terms of which we have interpreted the results of our computer 
experiments, viz., Equations (24), (25), and (28). The experiments themselves provided information that made it possible to check the validity of these approximations à posteriori. We turn now to a consideration of three of them.

\section{Gyrotropy}

The derivation of Equation (23) assumes that the particle distribution is very nearly gyrotropic. There were in fact small departures from gyrotropy in the measured $\langle f\rangle_{R}$. To check the validity of the gyrotropy approximation, the phase angle distribution function,

$$
h(\phi) \equiv \int_{\mu_{L}}^{\mu_{R}} \mathrm{~d} \mu\langle f\rangle_{\mathrm{R}}(\mu, \phi)
$$

was Fourier analyzed

$$
\mathrm{h}(\phi) \equiv \sum_{\mathrm{n}=-\infty}^{\infty} h_{\mathrm{n}} \mathrm{e}^{\mathrm{in} \phi},
$$

for each experiment. A hisiogram of $\mathrm{h}(\phi)$ for the $c=0$ of Figure 6 is given in Figure 8. The departure of $h$ from $h_{0}$ (dashed line) is evidently concentrated in the $\mathrm{n}= \pm 1$ modes and of small magnitude. The actual amplitude of the modulation in this case was measured to be

$$
\frac{\mid \mathrm{h}-\mathrm{h}_{\mathrm{o}} \mathrm{I}_{\max }}{\mathrm{h}_{\mathrm{o}}}=8.6 \times 10^{-3}
$$

which is in good agreement with the estimate derived in Paper I, viz.,

$$
\frac{\left|\mathrm{h}-\mathrm{h}_{\mathrm{o}}\right|}{\mathrm{h}_{\mathrm{o}}} \cong \frac{\left\langle\delta \omega^{2}\right\rangle}{\omega_{\mathrm{o}}^{2}}=10^{-2}
$$




\section{Boundary Conditions}

In deriving the boundary conditions, Equation (25), assumptions were made concerning the symmetry and statistical correlations of the probability distributions of $\phi$ and $\delta \omega$. To verify that these boundary conditions were actually satisfied in the experiments, we considered the equivalent Equations (B9), (B10), since each quantity appearing in the latter equations was directly determinable from the experimental data.

Thus, with $J$ and $\langle f\rangle_{R}$ determined in the manner described in Section IV-c, and IV $-\mathrm{d}$, and $\langle\alpha\rangle$ obtained from Equation (B10) and the relevant experimental parameters, the comparison for the case of Figure 6 is summarized in Table I. The close agreement between $J\langle f\rangle_{R}$ and $\langle\alpha\rangle$ at each boundary indicates that the boundary conditions, Equation (25), are indeed satisfied in the experiment.

\section{Adiabatic Approximation}

As already mentioned, a diffusive description of the behavior of $\langle f\rangle_{R}$ in the vicinity of the source appears to be invalid. Consequently, Equation (30) cannot be used there to determine the diffusion coefficient. In Section III-B, we discussed this difficulty from the Green's Function point of view. We here discuss the same breakdown from an alternative but equivalent.approach.

It is shown in Paper I that within the context of the partially-averaged field theory, the general steady state equation satisfied by $f_{0}$, the gyro-phase averaged distribution function averaged over the infinite ensemble is 


$$
\frac{\partial}{\partial \mu}\left\{\int_{0}^{2 \pi} \frac{\mathrm{d} \phi}{2 \pi}\left\langle\frac{\mathrm{du}}{\mathrm{dt}}\left\langle\Delta \mathrm{f}_{\mathrm{o}}\right\rangle_{\mathrm{P}}\right\rangle_{\mathcal{F}}\right\}=-\left(\frac{\mathrm{N}}{\Delta \mathrm{t}}\right) \delta\left(\mu-\mu_{\mathrm{S}}\right)
$$

where $\left\langle\Delta f_{0}\right\rangle_{p}$ is the asymptotic (in time) change in $f_{0}$ apparent to an observer moving from the point $\mu$ at $t=0$ along an orbit in the partially-averaged field $\langle\delta \mathrm{B}\rangle_{P}$, and \langle\rangle$_{\mathcal{F}}$ denotes a final ensemble average over the distribution of $\langle\delta \mathrm{B}\rangle_{\mathrm{p}}$.

If $f_{0}$ is expanded in a Taylor series about $\mu$, this change can be represented

$$
\left\langle\Delta \mathrm{f}_{\mathrm{o}}\right\rangle_{\mathrm{P}}=\frac{\partial \mathrm{f}_{\mathrm{o}}}{\partial \mu}\langle\Delta \mu\rangle_{p}+\mathrm{O}\left(\langle\Delta \mu\rangle_{p}^{2}\right)
$$

so that Equation (34) becomes

$$
\begin{gathered}
\frac{\partial}{\partial \mu}\left\{\left[\int_{0}^{2 \pi} \frac{\mathrm{d} \phi}{2 \pi}\left\langle\frac{\mathrm{d} \mu}{\mathrm{dt}}\langle\Delta \mu\rangle_{p}\right\rangle_{\Im \mathfrak{F}}\right] \frac{\partial \mathrm{f}_{\mathrm{o}}}{\partial \mu}+\mathrm{O}\left(\langle\Delta \mu\rangle_{p}^{2}\right)\right\} \\
=-\left(\frac{\mathrm{N}}{\Delta \mathrm{t}}\right) \delta\left(\mu-\mu_{\mathrm{S}}\right)
\end{gathered}
$$

which is of the same form as Equation (23) if higher order terms are neglected. Within the context of the partially-averaged field theory, use of Equation (36) neglecting $O\left(\langle\Delta \mu\rangle_{\rho}^{2}\right)$ terms to approximate Equation (34) is an adiabatic approximation similar to that made in standard quasi-linear theory.

Since the neglect of the higher order terms in expansion (36) is clearly invalid near the source, where $\partial \mathrm{f}_{\mathrm{o}} / \partial \mu$ is discontinuous, a diffusion equation like Equation (36) should not be expected to apply there.

Integration of Equations (34) and (36) from $\mu=\mu_{\mathrm{L}}$ to an interior point results in two different expressions for the flux: 


$$
\begin{gathered}
\mathrm{J}_{\text {non ad }}(\mu)=-\int_{0}^{2 \pi} \frac{\mathrm{d} \phi}{2 \pi}\left\langle\frac{\mathrm{d} \mu}{\mathrm{dt}}\left\langle\Delta \mathrm{f}_{\mathrm{o}}\right\rangle_{\mathcal{P}}\right\rangle_{\Im} \\
\mathrm{J}_{\mathrm{ad}}(\mu)=-\left[\int_{0}^{2 \pi} \frac{\mathrm{d} \phi}{2 \pi}\left\langle\frac{\mathrm{d} \mu}{\mathrm{dt}}\langle\Delta \mu\rangle_{\mathcal{P}}\right\rangle_{\Im}\right] \frac{\partial \mathrm{f}_{\mathrm{o}}}{\partial \mu}
\end{gathered}
$$

In regions where a diffusion description is valid these expressions should agree with each other and with the right side of Equation (30). $J_{\text {non ad }}$ and $J_{a d}$, will disagree where a diffusion picture, and thus Equations (36) and.(30), are inappropriate. For a given experiment these regions can be mapped by computing $J_{\text {non ad }}$ and $J_{a d}$ using the experimental distribution function $\langle f\rangle_{R}$ for $f_{0}$, and comparing. Such a comparison is shown in Figure 9 for the case of Figure 6 . It is evident from the figure that Equation (30) cannot be used to determine $\mathrm{D}_{\mu \mu}(\mu, \infty)$ in the region $0.2<\mu<0.65$. Also shown in Figure 9 is the flux observed in the experiment. The discrepancy between the computed flux and the observed flux is due to the fact that the partially-averaged field theory slightly overestimates the scattering at $\mu=0$, as is clear from Figure 7.

\section{RESULTS}

Steady state experiments like the one described in detail in Section IV were performed for a variety of values of the dimensionless parameters,

$$
\epsilon=\frac{\mathrm{z}_{\mathrm{c}} \omega_{\mathrm{o}}}{\mathrm{v}}
$$

which measures particle rigidity, and

$$
\eta=\frac{\left\langle\delta \omega^{2}\right\rangle^{3 / 2}}{\omega_{0}}
$$


which characterizes the strength of the random magnetic field. For the case of Figure 6, for example, these values were $\epsilon=1, \eta=0.1$.

Results of the experiments are given in Figures $10 \mathrm{am}$, where the dimensionless diffusion coefficient, $\left(z_{c} / v\right) D_{\mu \mu}(\mu, \infty)$, is plotted as a function of $\mu$ for each of the $(\epsilon, \eta)$ combinations tried. Also shown for comparison are the predictions of standard quasi-linear theory and partially-averaged field theory, represented by dashed and solid curves, respectively. The experimental parameters used in each of the experiments are given in Table $\Pi$.

As mentioned in Section IV-E, a velocity phase angle distribution function was compiled for each experiment to check the assumption of gyrotropy. In general, departures from cylindrical symmetry were concentrated in the $\mathrm{n}= \pm 1$ modes and had amplitudes in accordance with the theoretical estimate of Paper I,

$$
\frac{\left|h-h_{0}\right|}{h_{0}}=\eta^{2}
$$

A plot of $\max \left\{\left|\mathrm{h}-\mathrm{h}_{\mathrm{o}}\right|\right\} / \mathrm{h}_{\mathrm{o}}$ as a function of $\eta$ is given in Figure 11, with the estimate (39) superimposed for comparison. In no case was the departure from gyrotropy greater than $5 \%$.

Since the disagreement among the various theoretically computed diffusion coefficients is greatest at $\mu=0$, it is useful to investigate the manner in which $D_{\mu \mu}(0, \infty)$ scales with $\epsilon$ and $\eta$. The scaling observed in the experiments over roughly one decade of variation in each parameter is given in Figures 12a, b. From the figures it is clear that $D_{\mu \mu}(0, \infty)$ has a power law dependence on each 
parameter, with the exponent an increasing function of the other parameter, i..e.,

$$
\mathrm{D}_{\mu \mu}(0, \infty) \alpha \eta^{\mathrm{m}(\epsilon)} \epsilon_{\epsilon} \mathrm{q}(\eta)
$$

Least squares fits to the data give $\mathrm{m}(0.25)=2.5, \mathrm{~m}(\mathbf{1 . 0})=2.7, \mathrm{~m}(4.0)=3.1$, and $q(0.05)=0.18, q(0.10)=0.33, q(0.30)=0.62$. 
Table I

Data Needed to Check Satisfaction of Boundary Conditions for the Case of Figure 6

ㄴ.

\begin{tabular}{ccccc}
\hline$\mu$ & $\left(\mathrm{z}_{\mathrm{c}} / \mathrm{v}\right)|\mathrm{J}|$ & $\langle\mathrm{f}\rangle_{\mathrm{R}}$ & $\left(\mathrm{z}_{\mathrm{c}} / \mathrm{v}\right)|\mathrm{J}| /\langle\mathrm{f}\rangle_{\mathrm{R}}$ & $\left(\mathrm{z}_{\mathrm{c}} / \mathrm{v}\right)\langle\alpha\rangle$ \\
\hline-.65 & $+36.4 \pm \mathrm{I.1}$ & $854.3 \pm 62.8$ & $.043 \pm .003$ & .039 \\
+.65 & $219.6 \pm 1.1$ & $5319.1 \pm 137.1$ & $.041 \pm .001$ & .039 \\
\hline
\end{tabular}


Table II

Experimental Parameters Used in the Steady State Experiments of Figures 6 and 10

\begin{tabular}{ccccccccccc}
\hline Exp. & $\epsilon$ & $\eta$ & $\mathrm{R}$ & $\mathrm{N}$ & $\mu_{\mathrm{L}}$ & $\mu_{\mathrm{S}}$ & $\mu_{\mathrm{R}}$ & $\Delta \mu$ & $\mathrm{v} \Delta \mathrm{t} / \mathrm{z}_{\mathrm{c}}$ \\
\hline 1 & 0.25 & 0.05 & 400 & 10 & -.65 & .405 & .65 & .010 & .25 \\
2 & 0.25 & 0.10 & 400 & 32 & -.65 & .405 & .65 & .010 & .25 \\
3 & 0.25 & 0.30 & 800 & 20 & -.65 & .405 & .65 & .010 & .25 \\
4 & 1.00 & 0.05 & 100 & 80 & -.65 & .405 & .65 & .010 & .0625 \\
5 & 1.00 & 0.10 & 800 & 8 & -.65 & .405 & .65 & .010 & .03125 \\
6 & 1.00 & 0.30 & 800 & 8 & -.70 & .605 & .70 & .010 & .03125 \\
7 & 4.00 & 0.05 & 800 & 8 & -.50 & .3025 & .50 & .005 & .015625 \\
8 & 4.00 & 0.10 & 800 & 10 & -.65 & .405 & .65 & .010 & .015625 \\
9 & 4.00 & 0.30 & 800 & 16 & -.35 & .4025 & .55 & .005 & .00390625 \\
\hline
\end{tabular}




\section{REFERENCES}

1. T. J. Birmingham and F. C. Jones, Cosmic Ray Diffusion - Report of the Workshop in Cosmic Ray Diffusion Theory, NASA Technical Note, NASA TN D-7873 (1975).

2. F. C. Jones, T. J. Birmingham and T. B. Kaiser, Phys. Rev. A (1977).

3. A. M. Yaglom, An Introduction to the Theory of Stationary Random Functions (Prentice Hall, Englewood Cliffs, 1962).

4. M. C. Wang and G. E. Uhlenbeck, Rev. Mod. Phys. 17, 323 (1945).

5. J. R. Jokipii, Rev. Geophys. Space Phys. 9, 27 (1971).

6. F. C. Jones, T. J. Birmingham and T. B. Kaiser, Ap. J. 181, L139 (1973).

7. P. M. Morse and H. Feshbach, Methods of Theoretical Physics (McGrawHill, New York, 1953).

8. David Potter, Computational Physics (Wiley, London, 1973).

9. Philip R. Bevington, Data Reduction and Error Analysis for the Physical Sciences (McGraw-Hill, New York, 1969). 


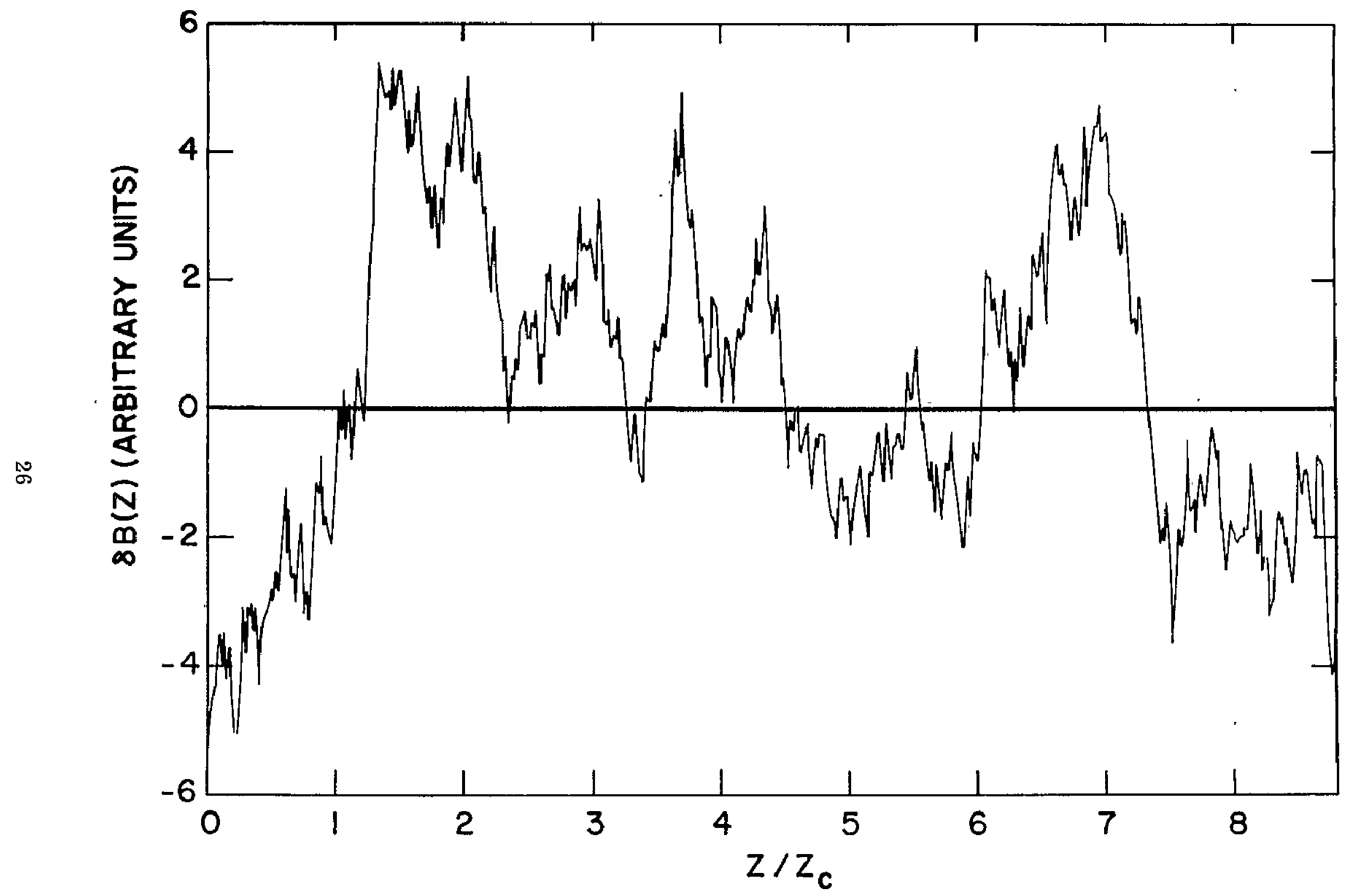

Figure 1. Segment of a typical realization of the random component of the magnetic field. 


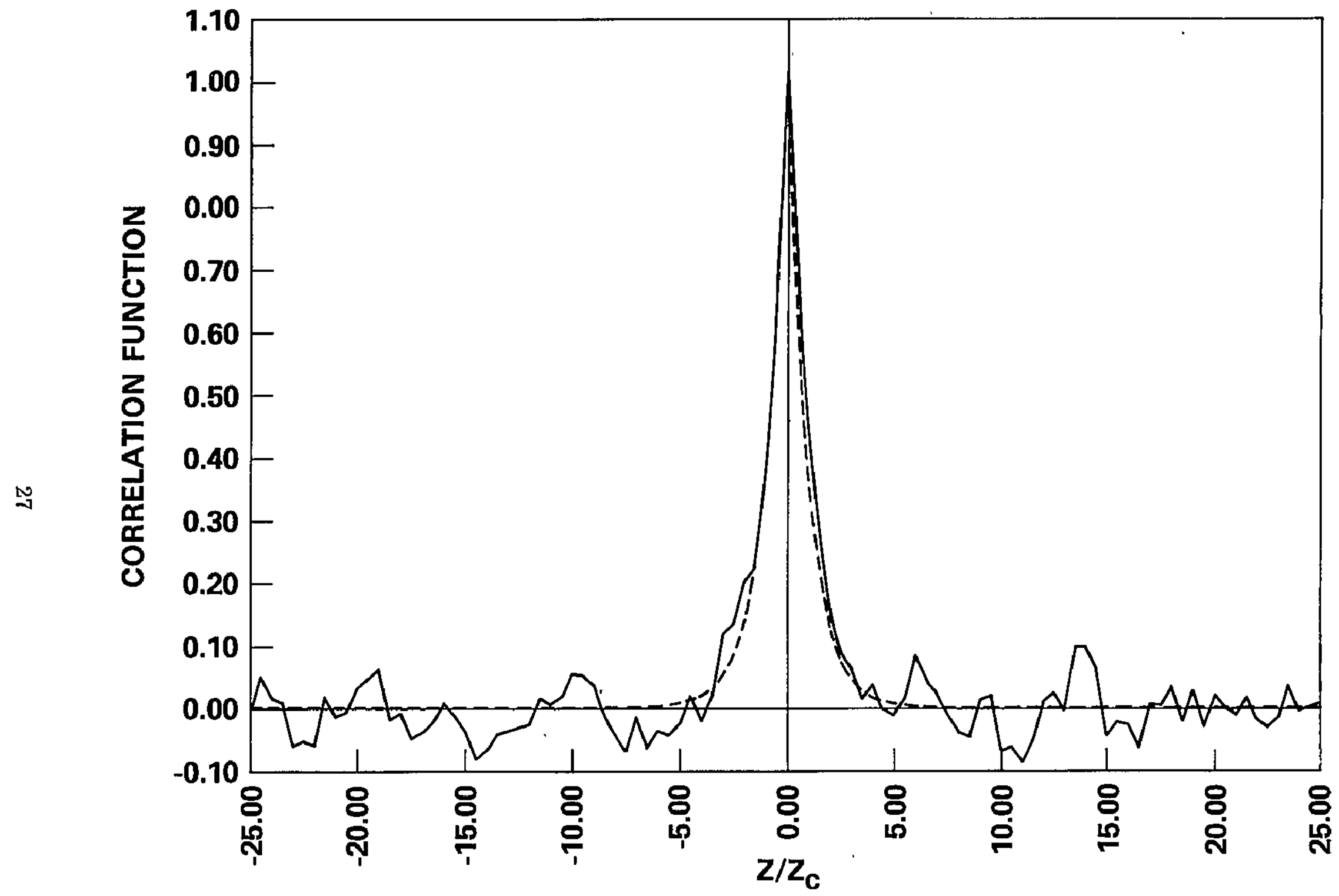

Figure 2. Empirical correlation function of $\delta \mathrm{B}(\mathrm{z})$ over the full ensemble of 800 realizations, Equation (4). The theoretical expression, Equation (2), is also plotted. 


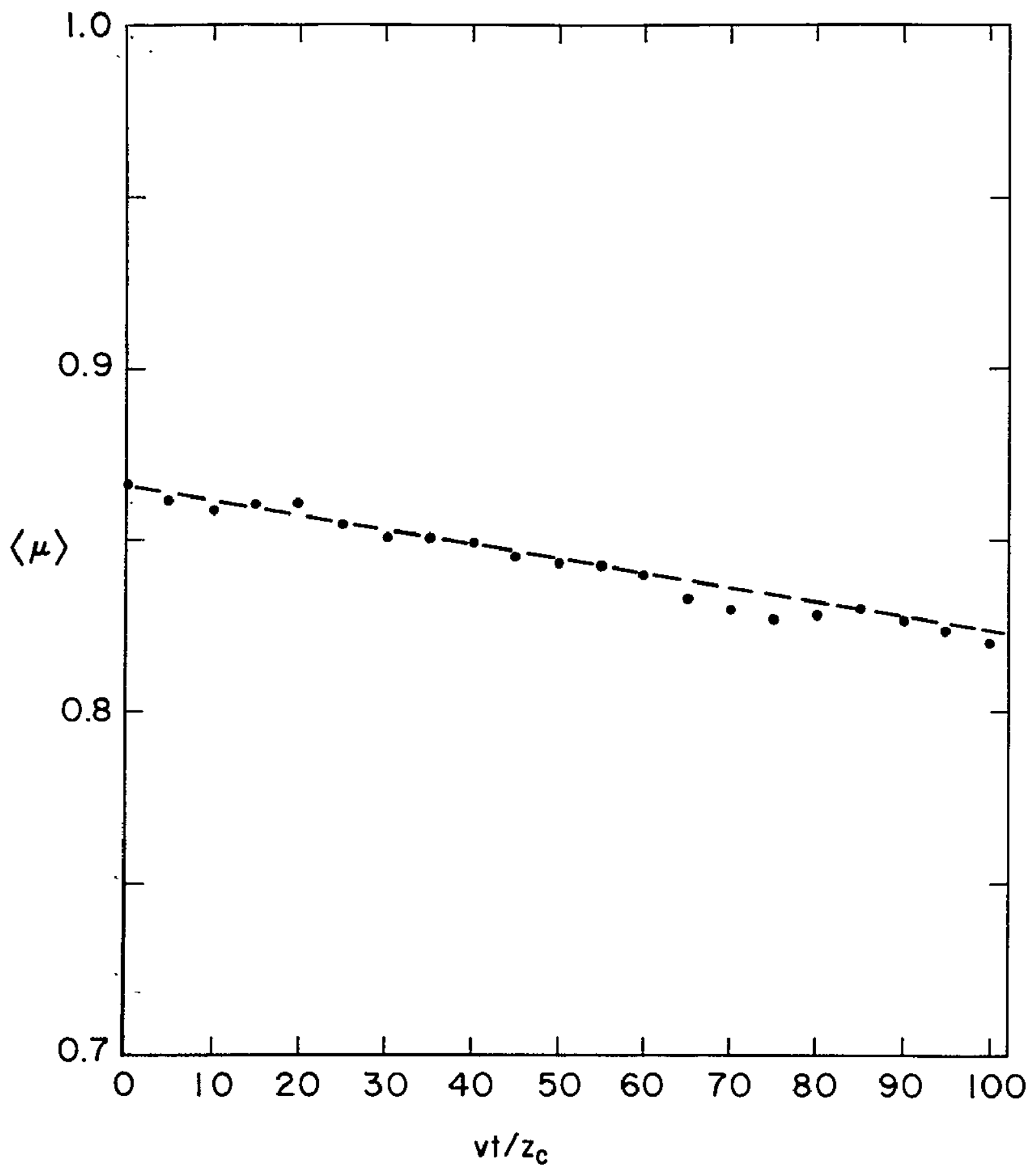

Figure 3. $\langle\mu(t)\rangle$ in a time dependent experiment with $\mathrm{z}_{\mathrm{c}} \omega_{\mathbf{0}} / \mathrm{v}=1, \mathrm{z}_{\mathrm{c}}\left\langle\delta \omega^{2}\right\rangle^{1 / 2} / \mathrm{v}=$ $10^{-3 / 2}, R=200, \mu_{0}=(3 / 4)^{1 / 2}$. Equation (19) is plotted for comparison. 


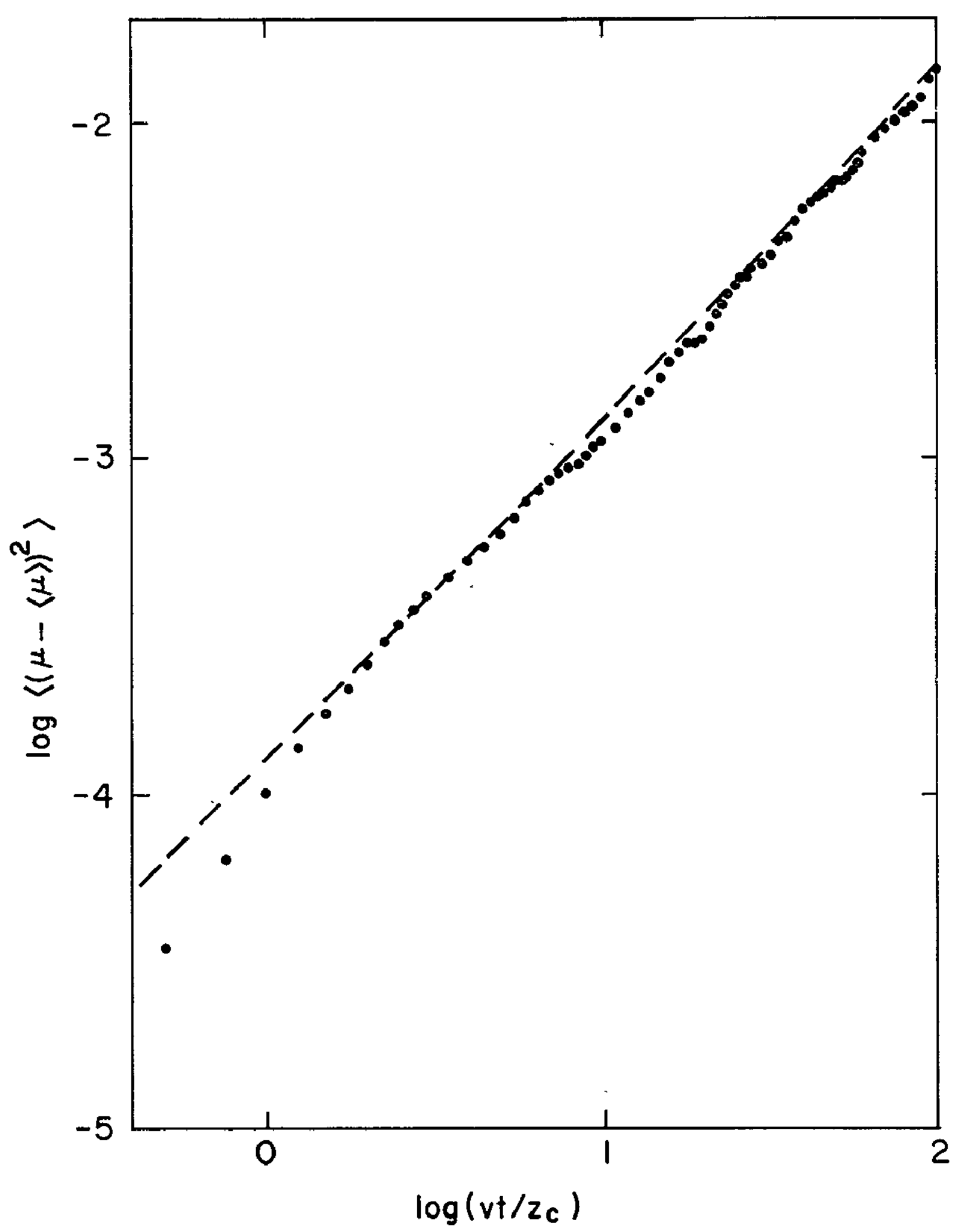

Figure 4. $\left\langle[\mu(t)-\langle\mu(t)\rangle]^{2}\right\rangle$ in the experiment of Figure 3, with Equation (21) plotted for comparison. 


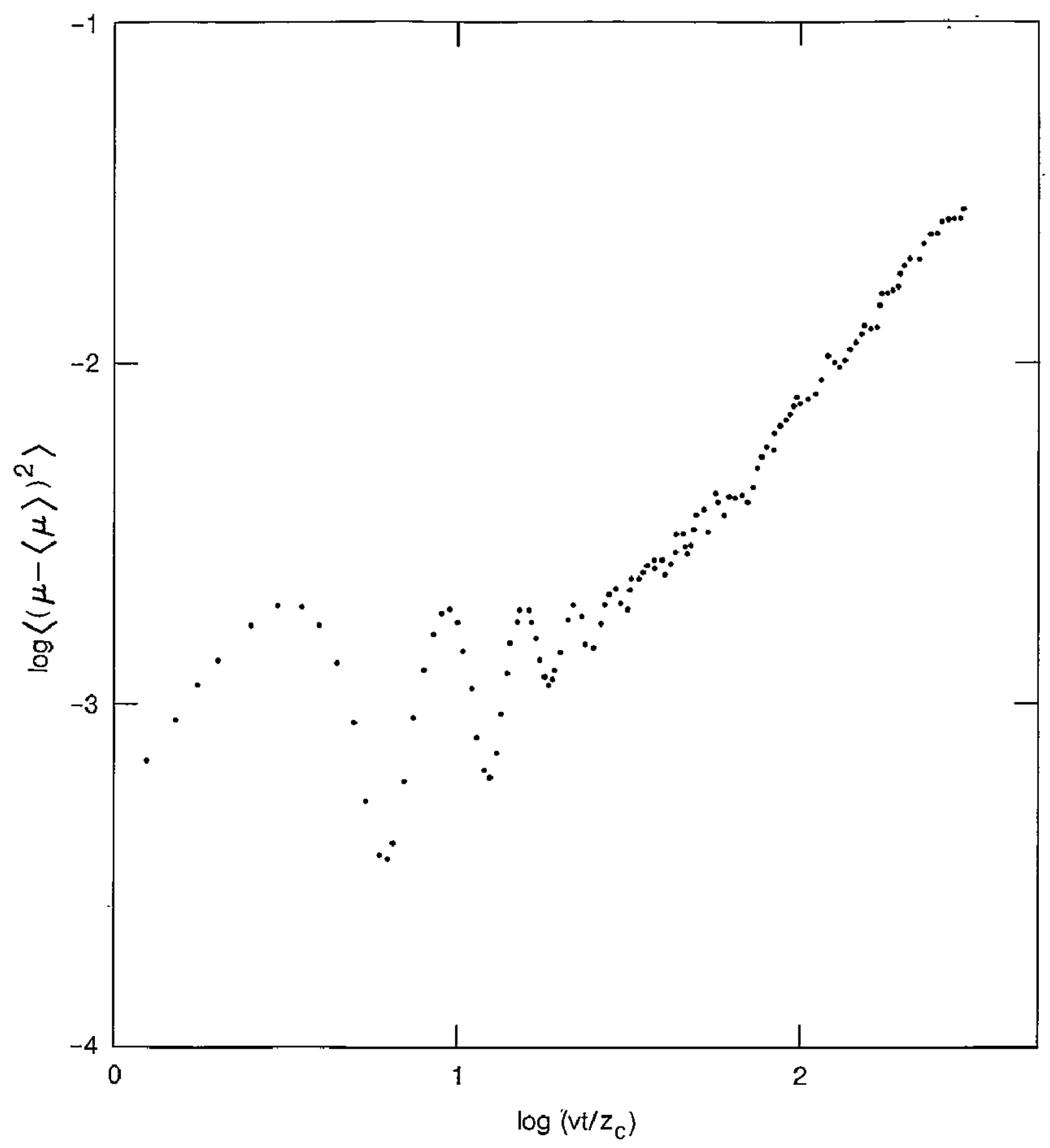

Figure 5. Same as Figure 4 except $\mu_{o}=0$. 


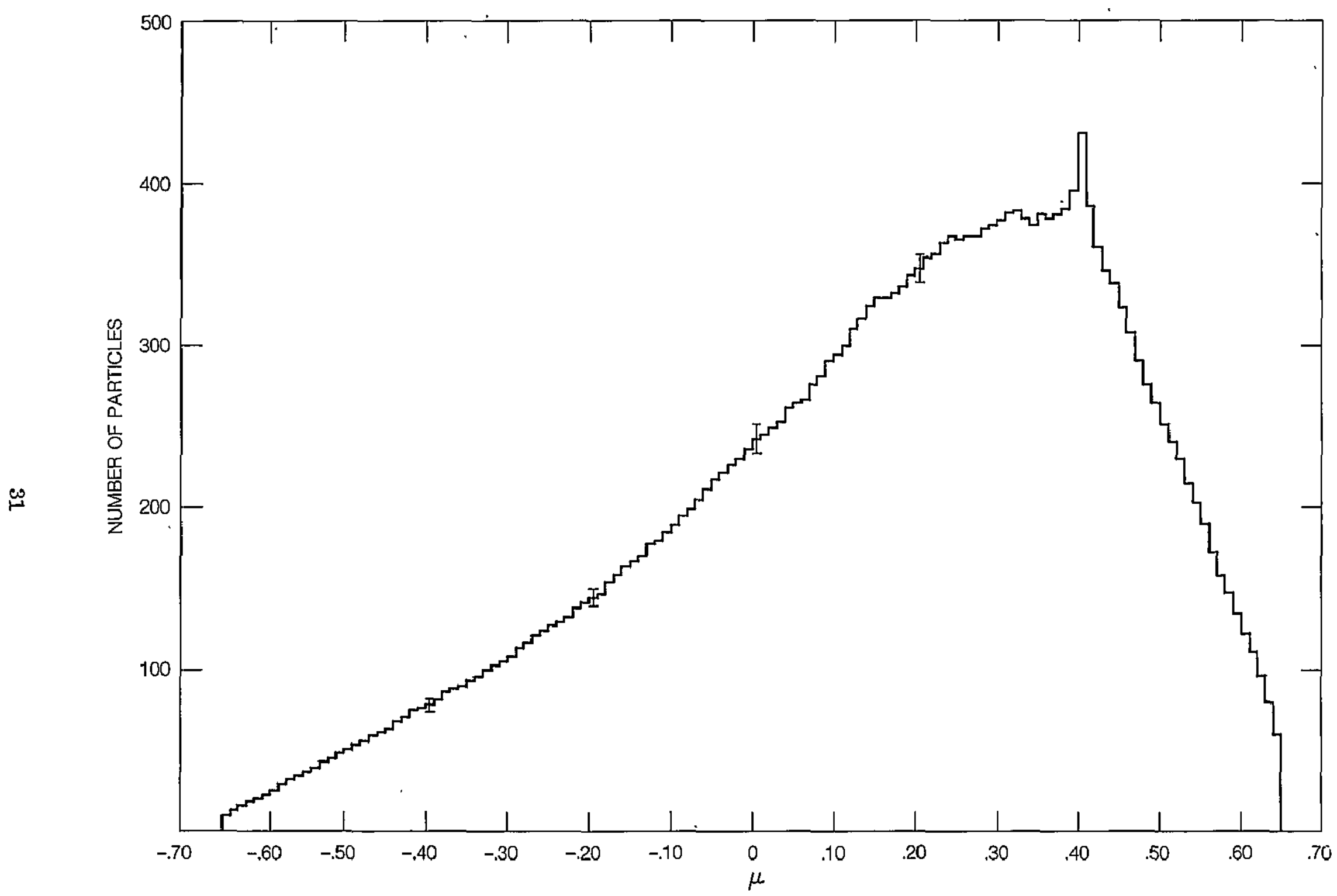

Figure 6. Histogram of $\mathrm{f}_{\mathrm{O}}(\mu, \infty)$ in a steady state experiment with $\mathrm{z}_{\mathrm{c}} \omega_{\mathrm{o}} / \mathrm{v}=1,\left\langle\delta \omega^{2}\right\rangle^{1 / 2} / \omega_{0}=.1$, $\mu_{\mathrm{L}}=-.65, \mu_{\mathrm{S}}=.405, \mu_{\mathrm{R}}=.65, \Delta \mu=.01, \mathrm{v} \Delta \mathrm{t} / \mathrm{z}_{\mathrm{c}}=2^{-5}, \mathrm{~N}=8, \mathrm{R}=800$. Error bäs are one-standard-deviation statistical uncertainties. 


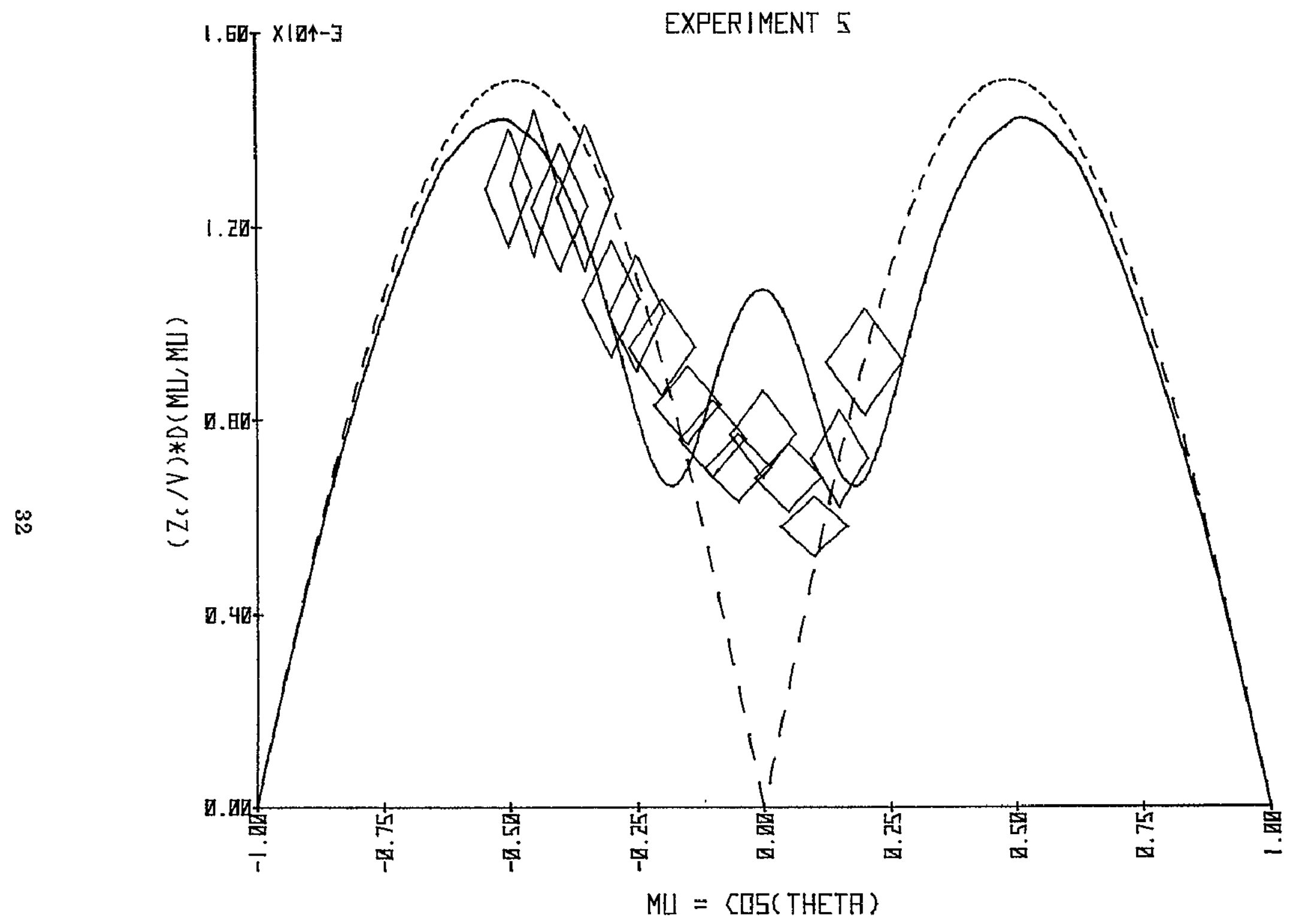

Figure 7. Dimensionless diffusion coefficient, $\left(\mathrm{z}_{\mathrm{c}} / \mathbf{v}\right) \mathbf{D}_{\mu \mu}(\mu, \infty)$, derived from the data of Figure 6 . Also shown are the diffusion coefficients derived in the partially averaged field theory (solid line) and standard quasi-linear theory (dashed line). 


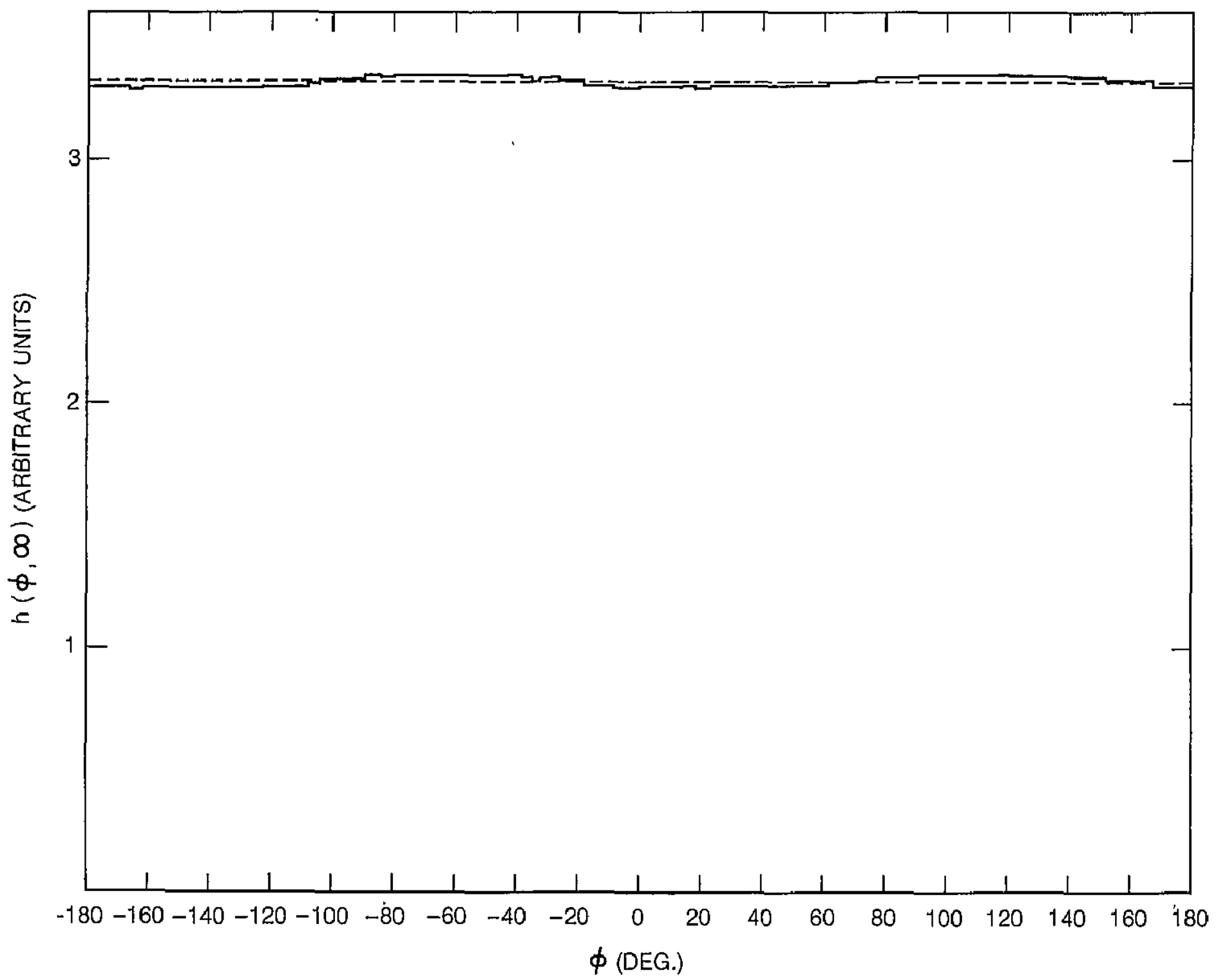

Figure 8. Histogram of the velocity phase angle distribution function, $\mathrm{h}(\phi, \infty)$, defined by Equation (33) for the experiment of Figure 6 . The gyrotropic component, $h_{0}$, is indicated by the dashed line. 


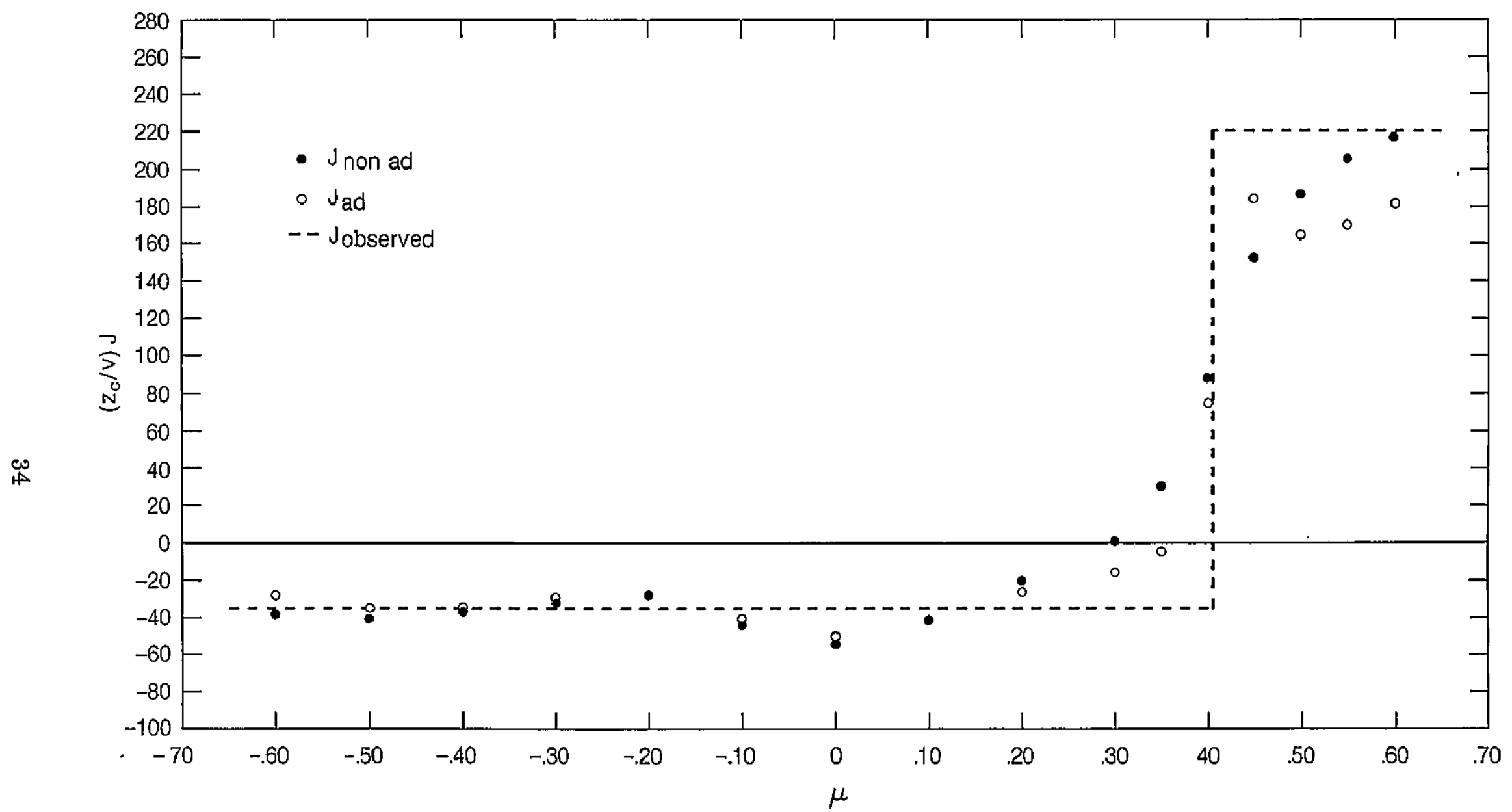

Figure 9. Flux in the experiment of Figure 6. Shown are $J_{\text {non ad }}$ Equation (38), $J_{\text {ad }}$ Equation (39), and: the observed $J_{L}$ and $J_{R}$. 


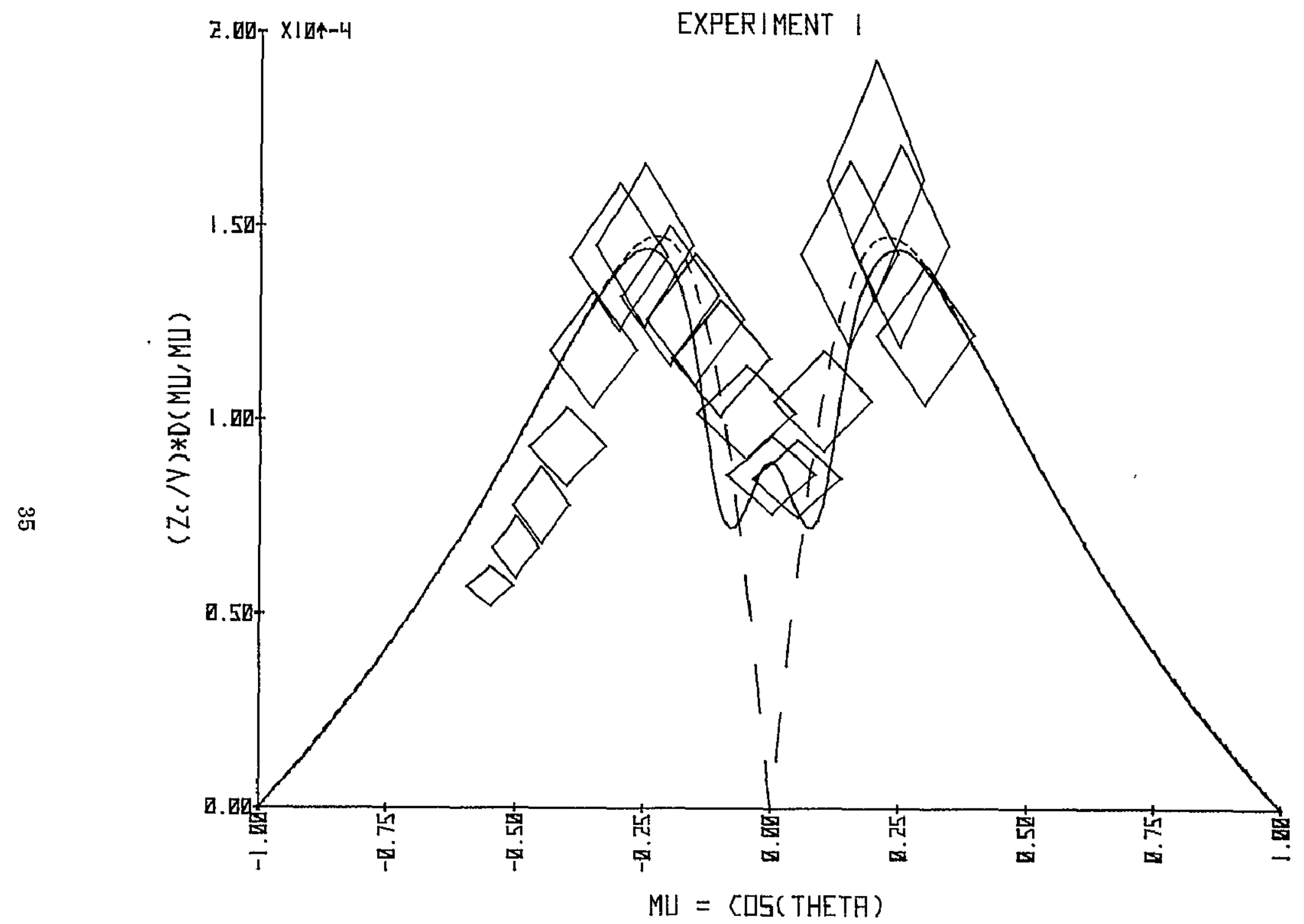

Figure 10a. Dimensionless diffusion coefficient, $(z \mathcal{H} / \mathrm{v}) \mathrm{D}_{\mu \mu}(\mu, \infty)$, derived from steady state experiments whose parameters are given in Table II. Also shown are the predictions of partiallyaveraged-field theory (solid line) and standard quasi-linear theory. (dashed line). 


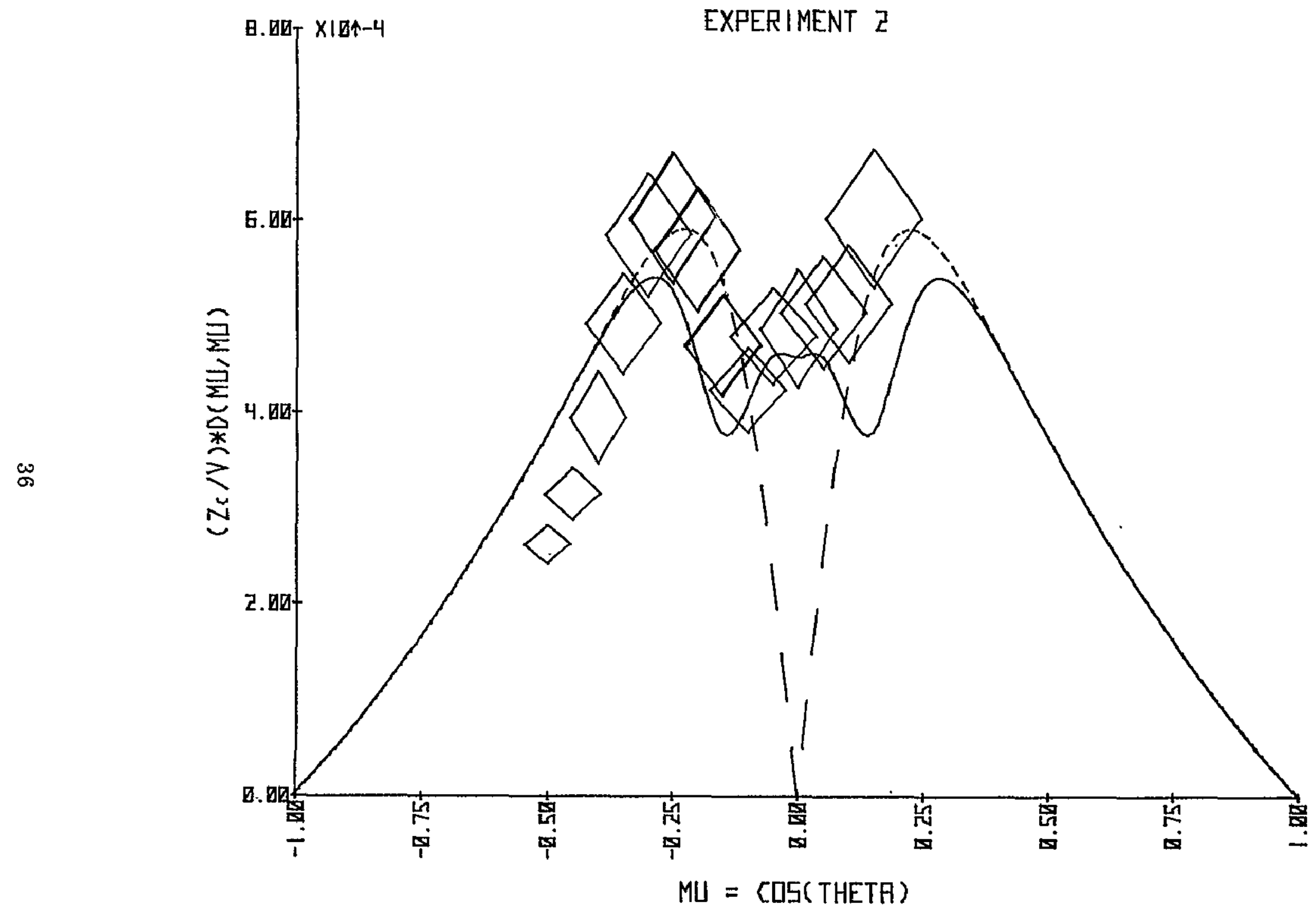

Figure 10b. 


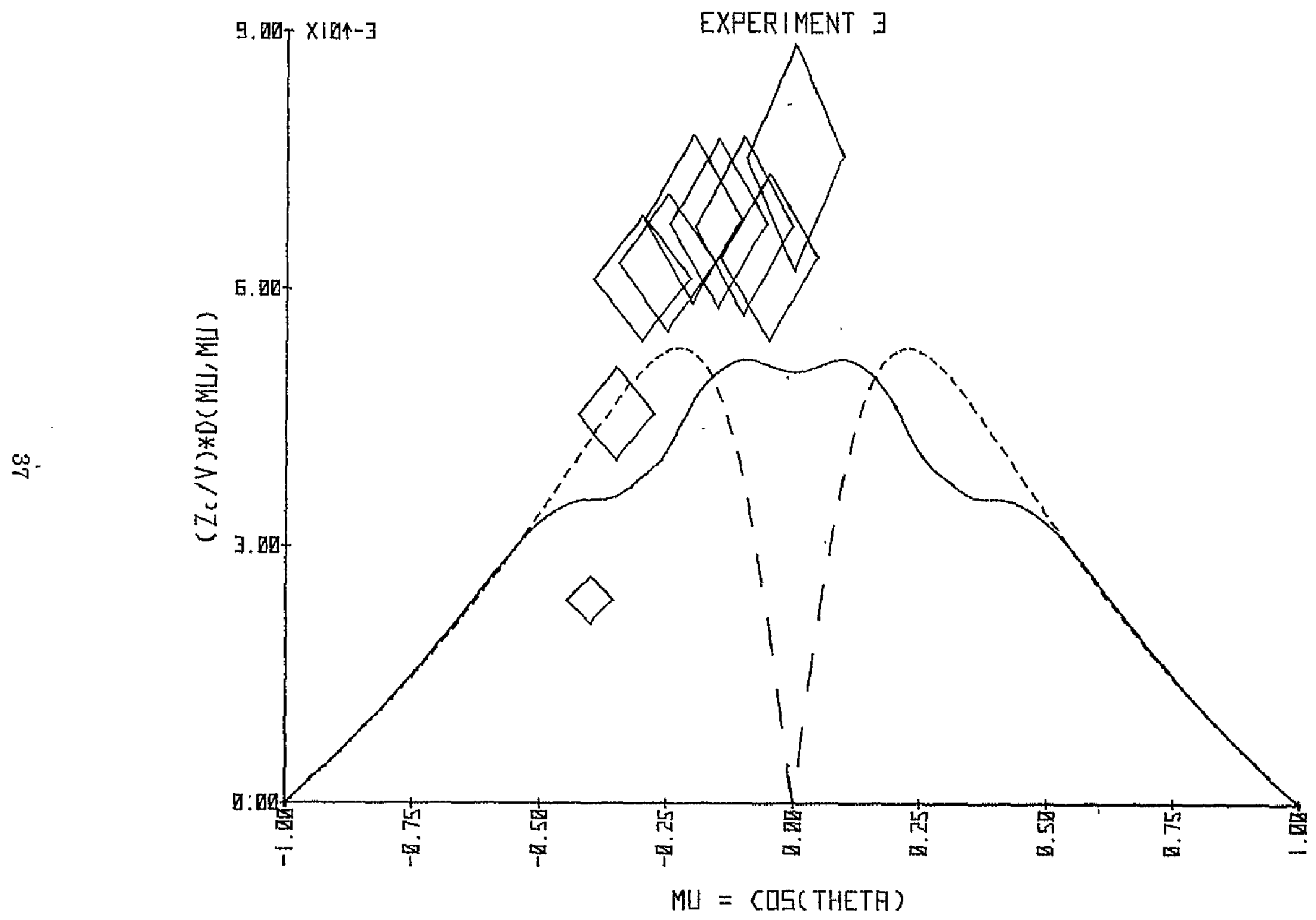

Figữe $10 \mathrm{c}$. 


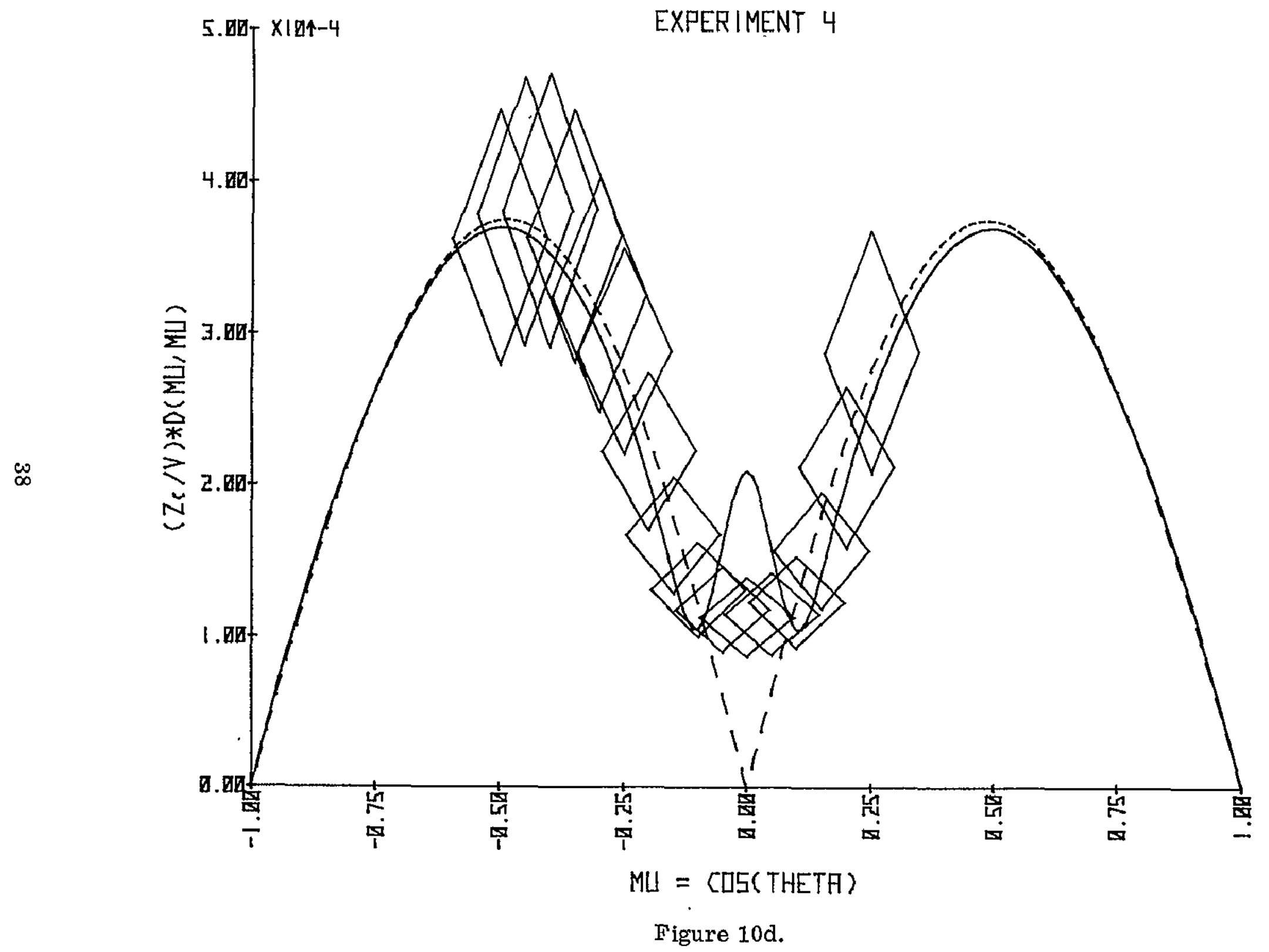




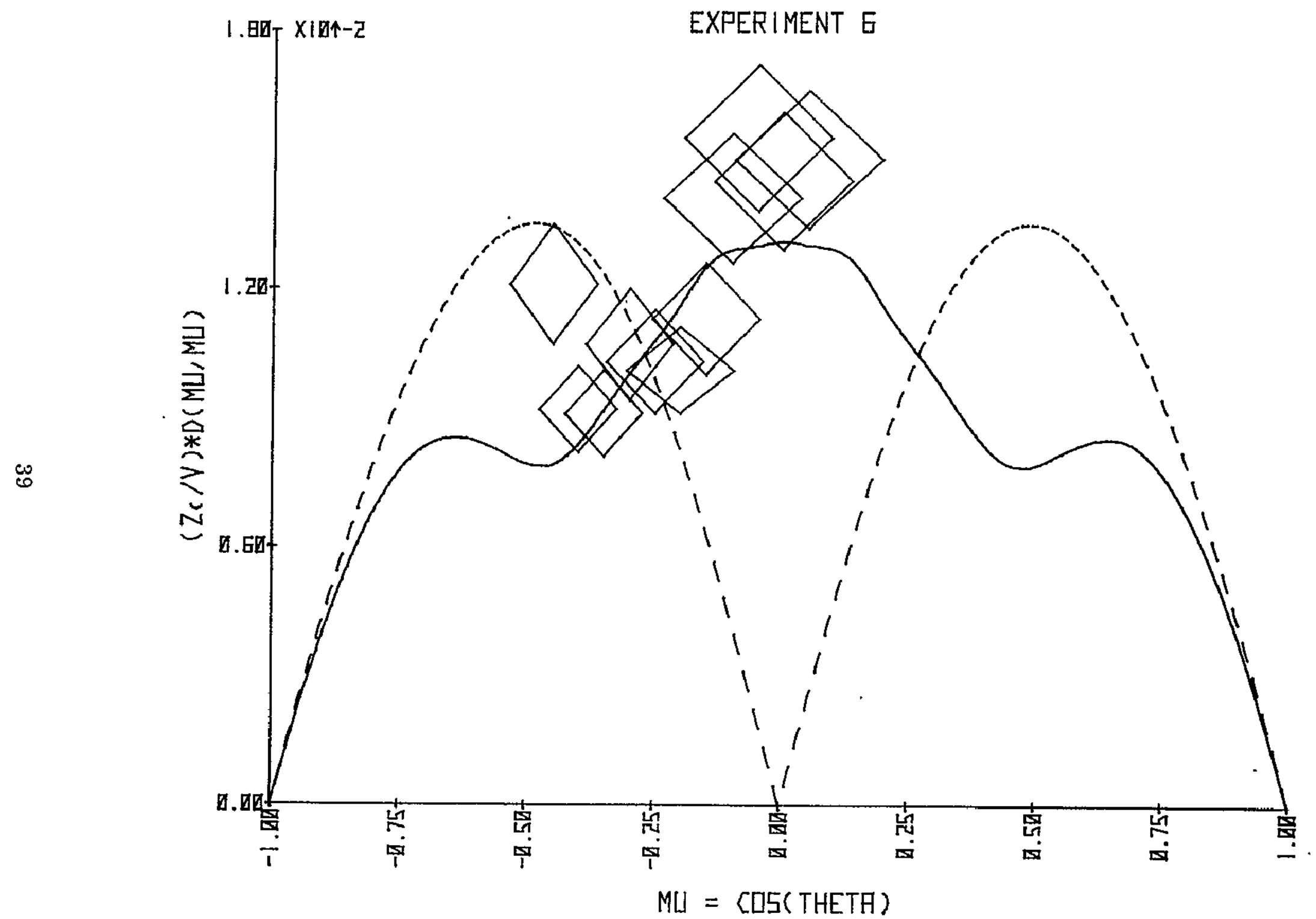

Figure 10e. 


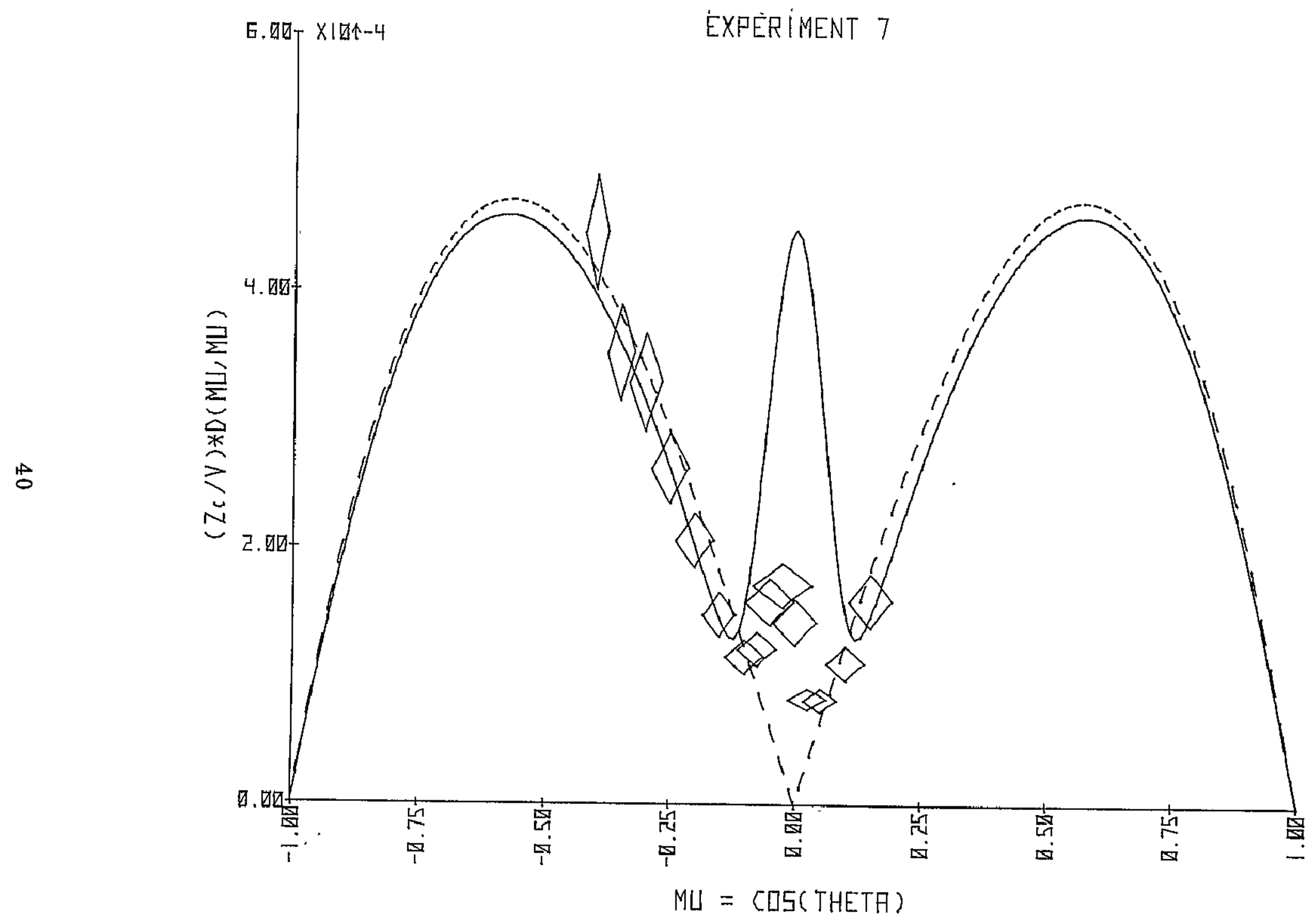

Figure $10 f$. 


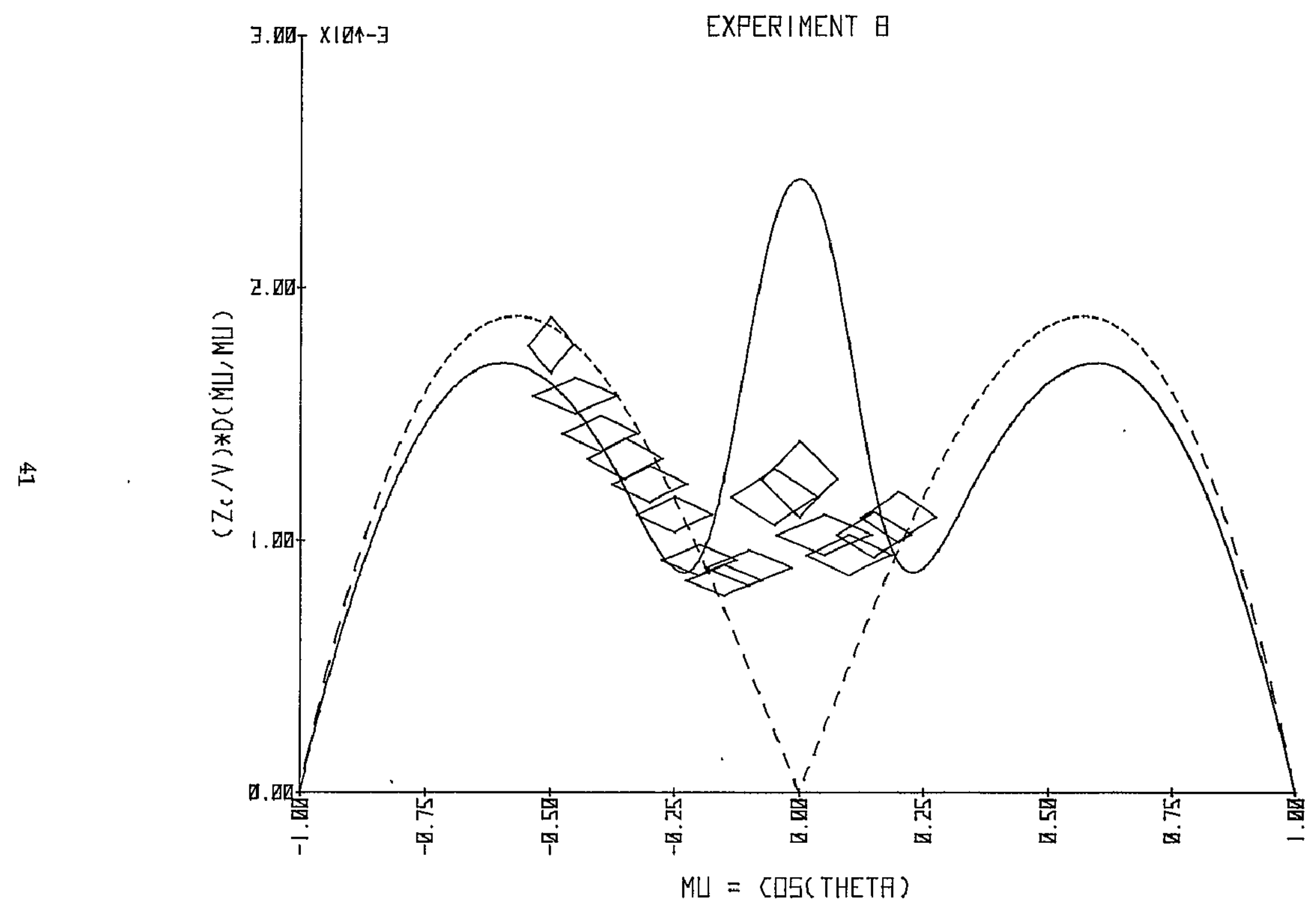

Figure 10g. 


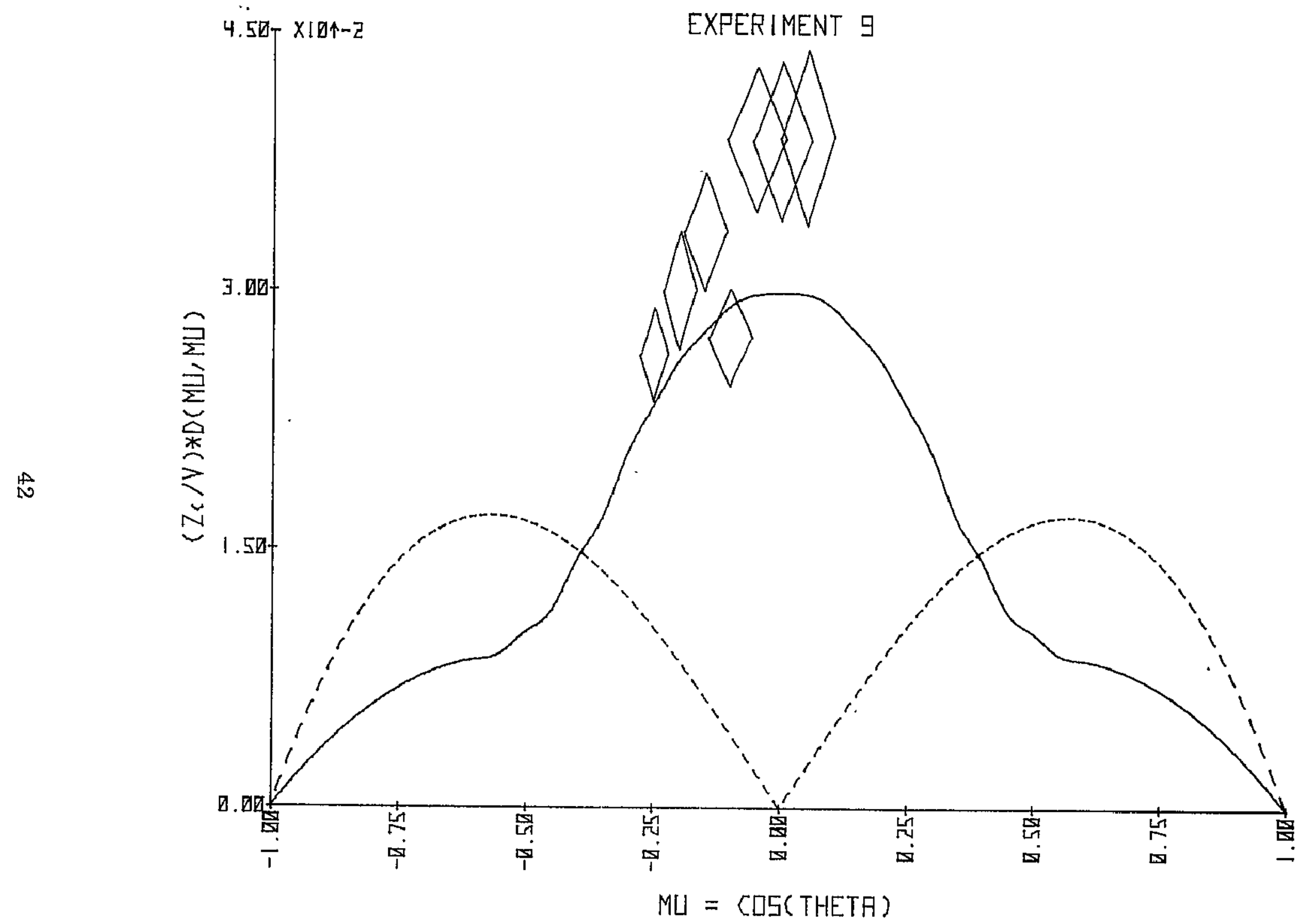

Figure 10h. 


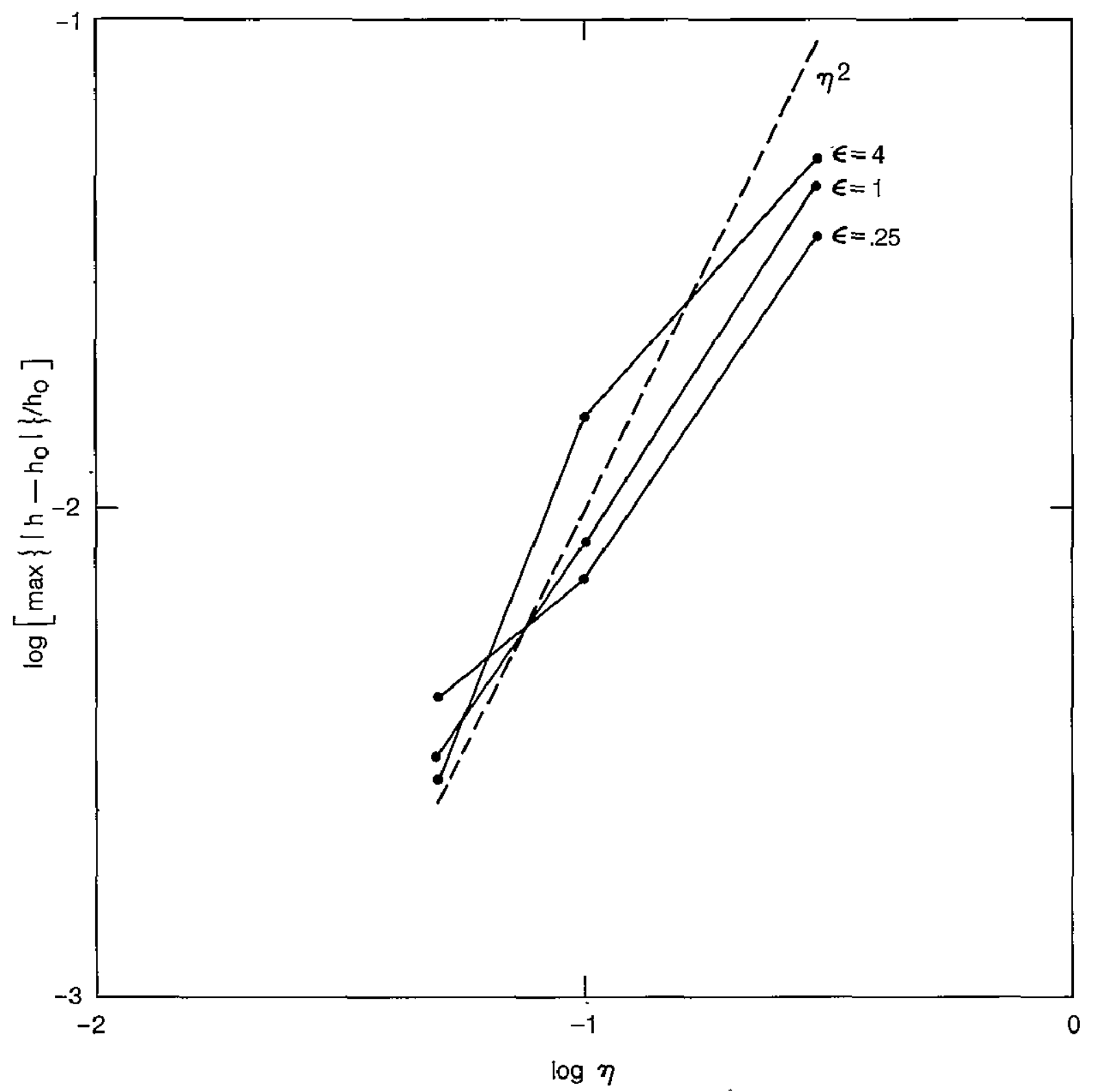

Figure 11. Plot of $\max \left\{\left|\mathrm{h}-\mathrm{h}_{\mathrm{o}}\right|\right\} / \mathrm{h}_{\mathrm{o}}$, which measures departure from gyrotropy, for each of the experiments of Table II. The theoretical estimate, Equation (40), is superimposed for comparison. 


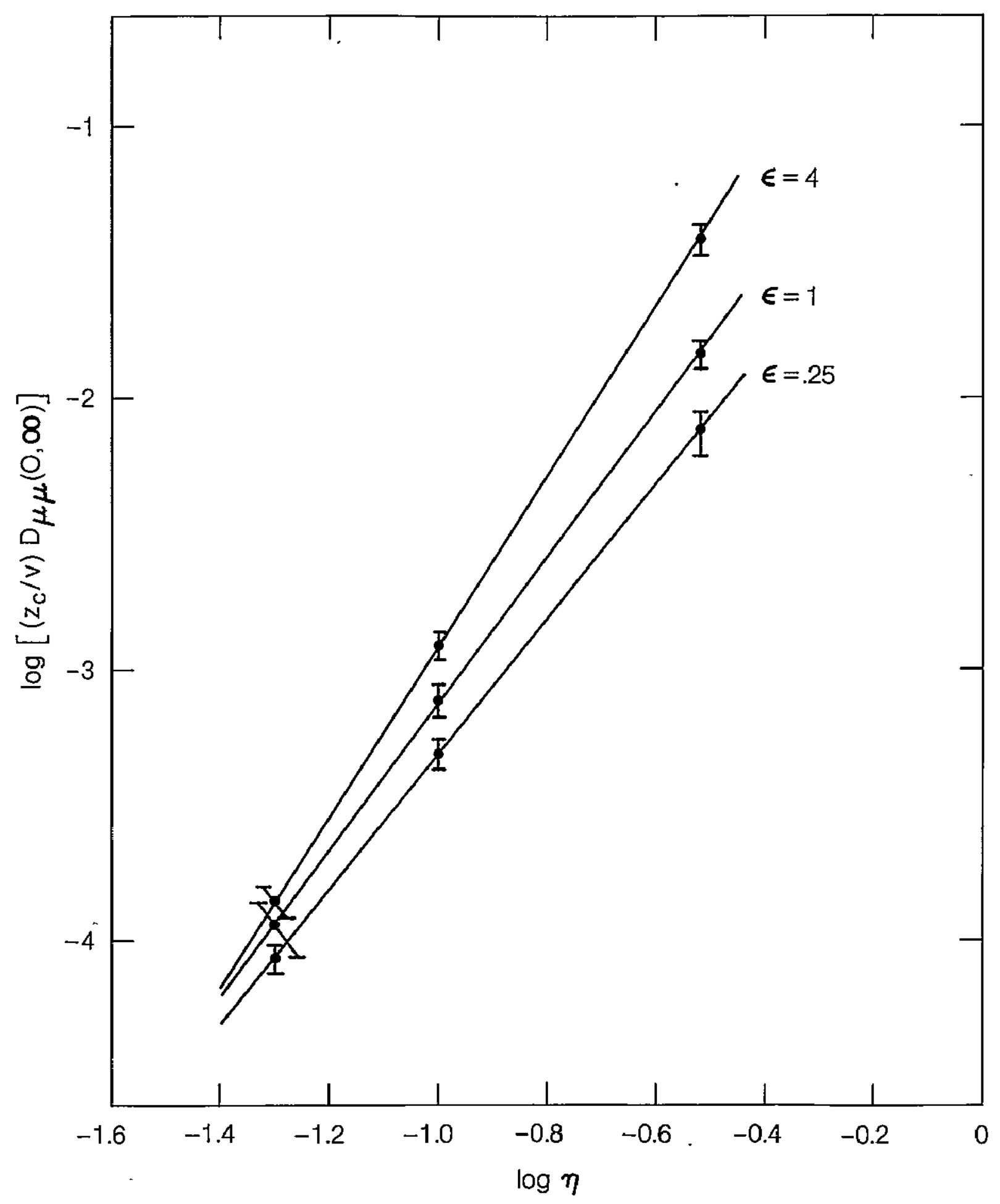

F igure 12a. Dimensionless diffusion coefficient at $\mu=0,\left(z_{c} / v\right) \quad D_{\mu \mu}(0, \infty)$, derived from the experiments of Table II. a) $\mathbf{D}_{\mu \mu}(0, \infty)$ as a function of $\eta$ for constant values of $\epsilon$. Solid lines are least squares fits. 


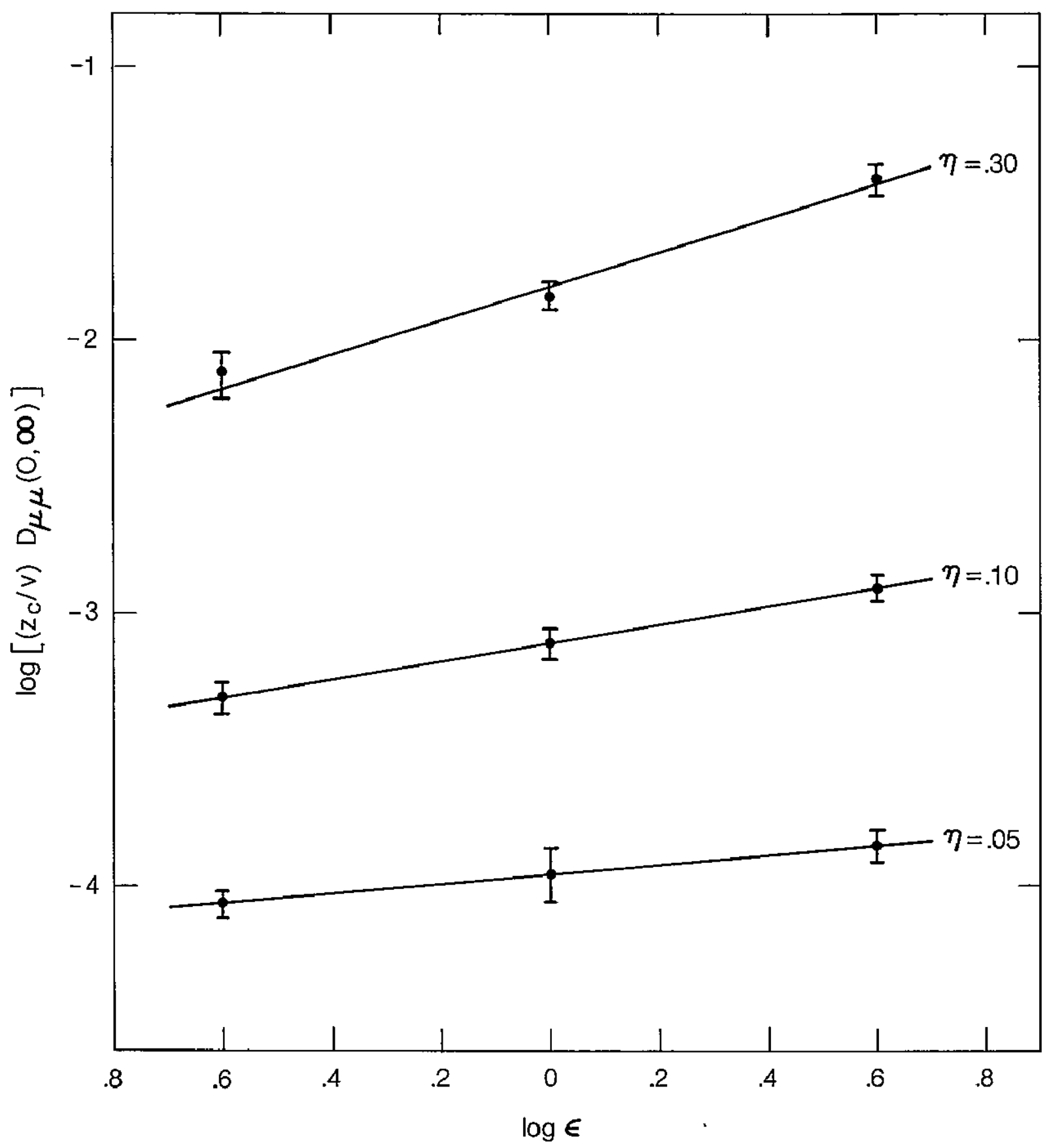

Figure 12b. Dimensionless diffusion coefficient at $\mu=0,\left(\mathrm{z}_{\mathrm{c}} / \mathrm{v}\right) \quad \mathrm{D}_{\mu \mu}(0, \infty)$, derived from the experiments of Table II. b) $D_{\mu \mu}(0, \infty)$ as a function of $\epsilon$. for constant values of $\eta$. Solid lines are least squares fits. 


\section{APPENDIX A \\ THE PARTICLE PUSHER}

\section{THE ALGORITHM}

The numerical scheme for integrating the particle equations of motion exploits the fact that for time increments, $t^{\prime}-t$, satisfying $\left|\omega(\underset{\sim}{x})\left(t^{\prime}-t\right)\right| \ll 1$, where $\omega(x)$ is the local gyrofrequency, the effect of a static magnetic field on a particle's velocity is simply to rotate it about the total field. Thus,

$$
\underset{\sim}{\mathrm{v}}\left(\mathrm{t}^{\prime}\right)=\underset{\approx}{\mathrm{T}}\left(\mathrm{t}, \mathrm{t}^{\prime}\right) \underset{\sim}{\mathrm{v}}(\mathrm{t})
$$

where the transformation matrix, $\underset{\approx}{\mathrm{T}}$, is a rotation.

Propagation in configuration space is accomplished by means of the secondorder implicit algorithm ${ }^{8}$

$$
\underset{\sim}{x}\left(t^{\prime}\right)=\underset{\sim}{x}(t)+\left[\underset{\sim}{v}(t)+\underset{\sim}{v}\left(t^{\prime}\right)\right] \frac{t^{\prime}-t}{2} .
$$

The remainder of this section is devoted to deriving the elements of the matrix $\underset{\approx}{\mathrm{T}}$ Consider two Cartesian coordinate systems, $\mathrm{S}$ and $\mathrm{S}^{\prime}$, whose z-axes are aligned respectively along the average and total magnetic fields. If a unit vector along the total field is denoted $\hat{b}$, and the coordinate unit vectors, $\widehat{e}_{i}$, $\hat{e}_{i}^{\prime}$, then $s^{\prime}$ is defined by

$$
\begin{aligned}
& \hat{e}_{3}^{\prime}=\hat{b} \\
& \hat{e}_{2}^{\prime}=\left(\hat{e}_{3} \times \hat{b}\right) / \mid \hat{e}_{3} \times \hat{b} l \\
& \hat{e}_{1}^{\prime}=\left(\hat{e_{3}} \times \hat{b}\right) \times \hat{b} /\left|\hat{e}_{3} \times \hat{b}\right|
\end{aligned}
$$


In $\mathrm{S}^{\prime}$ the velocity vector evolves according to

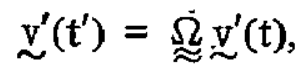

$$
\begin{aligned}
& \underset{\approx}{\approx}=\left[\begin{array}{ccc}
\cos \omega\left(t^{\prime}-t\right) & \sin \omega\left(t^{\prime}-t\right) & 0 \\
-\sin \omega\left(t^{\prime}-t\right) & \cos \omega\left(t^{\prime}-t\right) & 0 \\
0 & 0 & 1
\end{array}\right]
\end{aligned}
$$

The gyrofrequency, of course, depends on $\underset{\sim}{\mathrm{x}}$. It will be seen below that if the algorithm is to be reversible in time and maintain second order accuracy it is necessary that the position at which $\omega$ is evaluated be the intermediate point,

$$
\underset{\sim}{x_{1}}\left(t, t^{\prime}\right)=\underset{\sim}{x}(t)+\frac{1}{2} \underset{\sim}{\mathbb{v}}(t)\left(t^{\prime}-t\right)
$$

The transformation connecting $\mathrm{S}$ and $\mathrm{S}^{\prime}$ is also a rotation:

$$
\underline{\mathrm{v}}^{\prime}=\underset{\sim}{\mathrm{R}} \mathrm{v}
$$

In terms of the magnetic field components, $\underset{\approx}{\mathrm{R}}$ is

$$
\underset{\approx}{\mathrm{R}}=\left[\begin{array}{ccc}
\frac{\mathrm{B}_{1} \mathrm{~B}_{3}}{\mathrm{~B}_{1}} & \frac{\mathrm{B}_{2} \mathrm{~B}_{3}}{\mathrm{~B}_{1}} & -\frac{\mathrm{B}_{1}}{\mathrm{~B}} \\
-\frac{\mathrm{B}_{2}}{\mathrm{~B}_{\perp}} & \frac{\mathrm{B}_{1}}{\mathrm{~B}_{1}} & 0 \\
\frac{\mathrm{B}_{1}}{\mathrm{~B}} & \frac{\mathrm{B}_{2}}{\mathrm{~B}} & \frac{\mathrm{B}_{3}}{\mathrm{~B}}
\end{array}\right]
$$

where $B=|\underset{\sim}{B}|$ and $B_{\perp}=\left|\underset{\sim}{B}-\left(\underset{\sim}{B} \cdot \widehat{e}_{3}\right) \hat{e}_{3}\right|$.

If Equation (A.6) is used in Equation (A.3) and the result compared with Equation (A.1) it is clear that

$$
\underset{\approx}{\mathrm{T}}=\underset{\approx}{\mathrm{R}^{-1}} \underset{\approx}{\mathrm{R}}
$$


In terms of a vector, $\underset{\sim}{\omega}$,

$$
\underset{\sim}{\omega}=\omega \hat{b}
$$

and the functions

$$
\xi_{1}=\frac{\sin \omega\left(t^{\prime}-t\right)}{\omega\left(t^{\prime}-t\right)}, \quad \xi_{2}=2 \frac{1-\cos \dot{\omega}\left(t^{\prime}-t\right)}{\omega^{2}\left(t^{\prime}-t\right)^{2}}
$$

Thas the explicit form

$$
\begin{aligned}
& \mathrm{T}_{11}=1-\frac{1}{2} \xi_{2}\left(\omega_{2}^{2}+\omega_{3}^{2}\right)\left(\mathrm{t}^{\prime}-\mathrm{t}\right)^{2} \\
& \mathrm{~T}_{12}=\xi_{1} \omega_{3}\left(\mathrm{t}^{\prime}-\mathrm{t}\right)+\frac{1}{2} \xi_{2} \omega_{1} \omega_{2}\left(\mathrm{t}^{\prime}-\mathrm{t}\right)^{2} \\
& \mathrm{~T}_{13}=-\xi_{1} \omega_{2}\left(\mathrm{t}^{\prime}-t\right)+\frac{1}{2} \xi_{2} \omega_{1} \omega_{3}\left(t^{\prime}-t\right)^{2} \\
& T_{21}=-\xi_{1} \omega_{3}\left(t^{\prime}-t\right)+\frac{1}{2} \xi_{2} \omega_{1} \omega_{2}\left(t^{\prime}-t\right)^{2} \\
& \mathrm{~T}_{22}=1-\frac{1}{2} \xi_{2}\left(\omega_{1}^{2}+\omega_{3}^{2}\right)\left(t^{\prime}-t\right)^{2} \\
& T_{23}=\xi_{1} \omega_{1}\left(t^{\prime}-t\right)+\frac{1}{2} \xi_{2} \omega_{2} \omega_{3}\left(t^{\prime}-t\right)^{2} \\
& T_{31}=\xi_{1} \omega_{2}\left(t^{\prime}-t\right)+\frac{1}{2} \xi_{2} \omega_{1} \omega_{3}\left(t^{\prime}-t\right)^{2} \\
& T_{32}=-\xi_{1} \omega_{1}\left(t^{\prime}-t\right)+\frac{1}{2} \xi_{2} \omega_{2} \omega_{3}\left(t^{\prime}-t\right)^{2} \\
& \mathrm{~T}_{33}=1-\frac{1}{2} \xi_{2}\left(\omega_{1}^{2}+\omega_{2}^{2}\right)\left(\mathrm{t}^{\prime}-t\right)^{2}
\end{aligned}
$$


Although apparently complicated, the right sides of Equation (A.11) are well suited for numerical computation because the functions $\xi_{1}, \xi_{2}$ can be expanded in rapidly convergent power series in the small quantity $\omega\left(t^{\prime}-t\right)$. Consequently, the transformation involves only the operations of multiplication and addition; no quotients, square roots or other time-consuming operations need be performed.

The particle pushing scheme is completely specified by Equations (A.1), (A. 2), (A.4), (A.5), (A.7) and (A. 8). Various properties of the algorithm are described in the following section.

\section{PROPERTIES OF THE ALGORITHM}

a. Time Reversibility

Since the exact equations of motion of a charged particle in an electromagnetic field are reversible in time, it is desirable that the numerical integration scheme also possess this property. This is especially true in the present application, since the effect of irreversibility is a spurious diffusion of particle orbits.

To see that the algorithm is time-reversible, consider the effect of applying it twice in succession. A push from $t$ to $t^{\prime}$ is followed by one from $t^{\prime}$ to $t^{\prime \prime}=t$. The phase space position undergoes the transformations $(x, \underset{\sim}{\mathrm{v}}) \rightarrow\left(\mathrm{x}^{\prime}, \mathrm{v}_{\sim}^{\prime}\right) \rightarrow$ $\left(\mathrm{x}^{\prime \prime}, \stackrel{\mathrm{v}}{\prime}^{\prime \prime}\right)$. Time reversibility requires that $\left(\underset{\sim}{\mathrm{x}^{\prime \prime}}, \mathrm{v}^{\prime \prime}\right)=(\underset{\sim}{\mathrm{x}}, \mathrm{v})$. 
According to Equation (A.1), $\mathrm{v}^{\prime \prime}$ is related to $\mathrm{v}$ by

$$
\stackrel{\mathrm{V}^{\prime \prime}}{\sim} \underset{\approx}{\mathrm{T}}\left[\mathrm{x}_{\mathrm{I}}\left(\mathrm{t}^{\prime}, \mathrm{t}\right) ; \mathrm{t}^{\prime}, \mathrm{t}\right] \underset{\approx}{\mathrm{T}}\left[\mathrm{x}_{\mathrm{I}}\left(\mathrm{t}, \mathrm{t}^{\prime}\right) ; \mathrm{t}, \mathrm{t}^{\prime}\right] \underset{\sim}{\mathrm{v}}
$$

where the dependence of $\underset{\approx}{\mathrm{T}}$ on $\underset{\sim}{\mathrm{X}} \mathrm{I}$ has been indicated explicitly. A relation between $\underset{\sim}{x}$ and $\underset{\sim}{x}$ is obtained from Equation (A.2):

$$
\begin{aligned}
\underline{\sim}^{\prime \prime} & =x_{\sim}^{\prime}+\frac{1}{2}\left(\mathrm{y}^{\prime}+\mathrm{y}^{\prime \prime}\right)\left(\mathrm{t}-\mathrm{t}^{\prime}\right) \\
& =\underset{\sim}{\mathrm{x}}+\frac{1}{2}\left(\mathrm{v}+\mathrm{v}^{\prime}\right)\left(\mathrm{t}^{\prime}-\mathrm{t}\right)+\frac{1}{2}\left(\mathrm{v}^{\prime}+\mathrm{v}^{\prime \prime}\right)\left(\mathrm{t}-\mathrm{t}^{\prime}\right) \\
& =\underset{\sim}{\mathrm{x}}+\frac{1}{2}\left(\mathrm{v}-\mathrm{v}^{\prime \prime}\right)\left(\mathrm{t}^{\prime}-\mathrm{t}\right)
\end{aligned}
$$

From Equations (A.12) and (A.13) it is clear that the scheme is time reversible if

$$
\underset{\approx}{\mathrm{T}}\left[\underset{\sim}{\mathrm{x}}\left(\mathrm{t}^{\prime}, \mathrm{t}\right) ; \mathrm{t}^{\prime}, \mathrm{t}\right] \underset{\approx}{\mathrm{T}}\left[\mathrm{x}_{\mathrm{I}}\left(\mathrm{t}, \mathrm{t}^{\prime}\right) ; \mathrm{t}, \mathrm{t}^{\prime}\right]=\underset{\approx}{\mathrm{I}}
$$

Use of Equations (A.2) and (A.5) shows that $\underset{\mathrm{I}}{\mathrm{I}}\left(t^{\prime}, t\right)=\mathrm{x}_{\mathrm{I}}\left(t, t^{\prime}\right)$, i.e., that the forward and backward transformations both evaluate the magnetic field at the same point. This fact along with Equation (A.8) reduces Equation (A.14) to

$$
{\underset{\mathrm{R}}{-1}}^{\approx}\left(\mathrm{x}_{\mathrm{I}}\right) \underset{\approx}{\Omega}\left(\mathrm{t}-\mathrm{t}^{\prime}\right) \underset{\approx}{\Omega}\left(\mathrm{t}^{\prime}-\mathrm{t}\right) \underset{\approx}{\mathrm{R}}\left(\mathrm{x}_{\mathrm{I}}\right)=\mathrm{I}
$$

which use of the definition (A. 4) shows to be identically true. Therefore the algorithm is, in fact, exactly reversible in time.

b. Conservation of Energy

Another property of the exact equations of motion of a particle in a static magnetic field is the conservation of kinetic energy. That energy is conserved 
by the numerical scheme follows immediately from the fact that the kinetic energy is proportional to $|\underset{\sim}{\mathrm{v}}|^{2}$, which is invariant under the orthogonal transformation, $\underset{\approx}{\mathrm{T}}$.

c. Accuracy

An important consideration in any numerical approximation is its accuracy. In this section it will be shown that the accuracy of the particle pushing algorithm is of second order in the time step.

$$
\begin{aligned}
& \text { Let } \underset{\sim}{x}=\underset{\sim}{x}(t),{\underset{\sim}{x}}^{\prime}=\underset{\sim}{x}\left(t^{\prime}\right), \underset{\sim}{v}=\underset{\sim}{v}(t),{\underset{\sim}{v}}^{\prime}=\underset{\sim}{v}\left(t^{\prime}\right) \text { and } \Delta t=t^{\prime}-t \text {. Then } \\
& \mathrm{v}^{\prime}=\underset{\sim}{\mathrm{v}}+\frac{\mathrm{dv}}{\mathrm{dt}} \Delta \mathrm{t}+\frac{1}{2} \frac{\mathrm{d}^{2} \mathrm{v}}{\underset{\sim}{\sim} t^{2}} \Delta \mathrm{t}^{2}+\mathrm{O}\left(\Delta \mathrm{t}^{3}\right)
\end{aligned}
$$

In terms of the matrix

$$
\underset{\approx}{A}(x)=\left[\begin{array}{ccc}
0 & \omega_{3}(\underset{\sim}{(x)} & -\omega_{2}(\underset{\sim}{(x)} \\
-\omega_{3}(\underset{\sim}{(x)} & 0 & \omega_{1} \underset{\sim}{(x)} \\
\omega_{2}(\underset{\sim}{(x)} & -\omega_{1}(\underset{\sim}{(x)} & 0
\end{array}\right]
$$

the particle acceleration is

$$
\frac{\mathrm{d} y}{\mathrm{dt}}=\underset{\approx}{\mathrm{A}} \mathrm{v}
$$

With this, Equation (A.15) can be put in the form

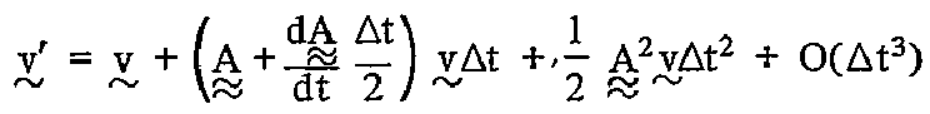


Now the value of $\underset{\approx}{\mathrm{A}}$ at the intermediate point, $\underset{\mathrm{I}}{\mathrm{x}}=\underset{\sim}{\mathrm{x}}+\frac{1}{2} \mathrm{v} \Delta t$, is.

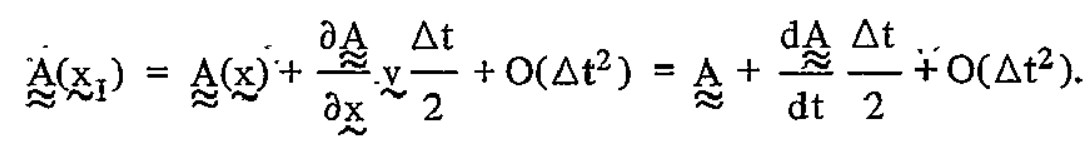

Furthermore,

$$
\underset{\approx}{\mathrm{A}}\left(\mathrm{x}_{\mathrm{I}}\right)^{2}=\underset{\approx}{\mathrm{A}}(\mathrm{x})^{2}+\mathrm{O}(\Delta \mathrm{t})
$$

Therefore, Equation (A.18) can be written

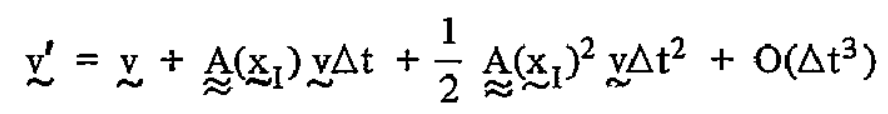

Since, by the definitions (A.10),

$$
\xi_{1}\left(x_{I}\right)=\xi_{2}\left(x_{I}\right)=1+O\left(\Delta t^{2}\right)
$$

it is valid to write Equation $(A .19)$ as

$$
\underline{\sim}^{\prime}=\left[\underset{\approx}{I}+\xi_{1}\left(x_{I}\right) \underset{\approx}{A}\left(x_{I}\right) \Delta t+\frac{1}{2} \xi_{2}\left(\underset{\sim}{x_{I}}\right) \underset{\approx}{A}\left(x_{I}\right)^{2} \Delta t^{2}\right] \underset{\sim}{\mathrm{z}}+O\left(\Delta t^{3}\right)
$$

Reference to Equation (A.11) shows that the matrix in square brackets in Equation (A.20) is precisely $\underset{\approx}{\mathrm{T}}$. Therefore, the truncation error of the velocity integration algorithm is third order in the time step.

It is easily seen that the same is true in position space. The Taylor series for $x^{\prime}$ is

$$
\begin{aligned}
\underset{\sim}{x^{\prime}} & =\underset{\sim}{x}+\frac{d \underset{\sim}{d}}{d t} \Delta t+\frac{1}{2} \frac{d^{2} \underset{\sim}{\sim}}{d t^{2}} \Delta t^{2}+O\left(\Delta t^{3}\right) \\
& =\underset{\sim}{x}+\underset{\sim}{v} \frac{\Delta t}{2}+\left(\underset{\sim}{v}+\frac{d v}{d t} \Delta t\right) \frac{\Delta t}{2}+O\left(\Delta t^{3}\right) \\
& =\underset{\sim}{x}+\left(\underset{\sim}{v}+\underline{\sim}^{\prime}\right) \frac{\Delta t}{2}+O\left(\Delta t^{3}\right)
\end{aligned}
$$

which is identical to Equation (A.2) through second order terms. 


\section{d. Computational Speed}

In practice, the functions $\xi_{1}$ and $\xi_{2}$ are expanded in power series in $\omega \Delta t$ up to and including second order terms. This maintains second order accuracy in $\underset{\sim}{\mathrm{x}}$ and $\underset{\sim}{\mathrm{V}}$ and time-reversibility and energy conservation to $\mathrm{O}\left[(\omega \Delta \mathrm{t})^{5}\right]$.

In the special case of a slab model magnetic field, where

$$
\underset{\sim}{\omega}(x)=\hat{e}_{1} \delta \omega\left(\underset{\sim}{x} \cdot \hat{e}_{3}\right)+\hat{e}_{3} \omega_{0}
$$

the computation time required by an IBM $360 / 91$ computer to push a particle's phase space position $(\underset{\sim}{\mathbb{v}}, \underset{\sim}{\mathrm{V}})$ through one integration step is $25 \mu$ sec. This includes a table look-up with linear intepolation to evaluate $\underset{\sim}{\omega(x)}$ and accumulation of the velocity distribution function. A typical simulation experiment involving $10^{4}$ particles, followed for an average of 2000 time steps each, thus requires about 8 minutes of cpu time. 


\section{APPENDIX B}

BOUNDARY CONDITIONS FOR THE STEADY STATE EXPERIMENT

Let $\alpha \equiv \mathrm{d} \mu / \mathrm{d} t$ and $\Psi(\mu, \alpha)$ be the joint probability density for a particle to be found in the incremental range $\{(\mu, \alpha),(\mu+\mathrm{d} \mu, \alpha+\mathrm{d} \alpha)\}$. Then the flux of particles in $\mu$-space is

$$
\mathrm{J}(\mu)=\int_{-\infty}^{\infty} \mathrm{d} \alpha \alpha \Psi(\mu, \alpha)
$$

In order to relate $\Psi$ to $f_{0}(\mu)$, the ensemble averaged distribution function, we also define the conditional probability density for $\alpha$, given $\mu, \psi(\alpha \mid \mu)$. Thus

$$
\Psi(\mu, \alpha) \equiv \mathrm{f}_{\mathrm{o}}(\mu) \psi(\alpha \mid \mu)
$$

so that

$$
\mathrm{J}(\mu)=\mathrm{f}_{\mathrm{o}}(\mu) \int_{-\infty}^{\infty} \mathrm{d} \alpha \alpha \psi(\alpha \mid \mu) \equiv \mathrm{f}_{\mathrm{o}}(\mu)\langle\alpha(\mu)\rangle
$$

When $\mu$ is an absorbing boundary $\mu=\mu_{\mathrm{B}}$, the conditional probability, $\psi\left(\alpha \mid \mu_{B}\right)$, can be estimated as follows. In a magnetic field of the form (1), Newton's equation for $\alpha$ is

$$
\alpha=\frac{\mathrm{d} \mu}{\mathrm{dt}}=-\left(1-\mu^{2}\right)^{1 / 2} \delta \omega \sin \phi
$$

where $\delta \omega=\mathrm{q} \delta \mathrm{B}(\gamma \mathrm{mc})^{-1}$ and $\phi$ is the gyrophase angle $(-\pi \leqslant \phi \leqslant \pi)$. For definiteness, consider a boundary to the right of the source, $\mu_{\mathrm{B}}=\mu_{\mathrm{R}}>\mu_{\mathrm{S}}$. Then, since the boundary is perfectly absorbing, there can be no particles with $\alpha<0$, in which case Equation (B. 4) implies that $\delta \omega$ and $\phi$ must have opposite algebraic 
signs. Furthermore, the distribution of $\delta \omega$ (regardless of $\phi$ ) is Gaussian, ${ }^{2}$ and, hence, symmetric about $\delta \omega=0$, and the distribution of $\phi$ (regardless of $\delta \omega)$ is uniform, ${ }^{2}$ and therefore symmetric about $\phi=0$. Assuming that this symmetry holds for the conditional distribution of $\delta \omega$ (given $\phi$ ) and $\phi$ (given $\delta \omega)$, i. e., that for every particle with $(\delta \omega, \phi)$ there is one with $(-\delta \omega,-\phi)$, we can equivalently take

$$
\alpha=\left(1-\mu_{\mathrm{R}}^{2}\right)^{1 / 2} \chi^{\sigma}
$$

where

$$
\begin{array}{ll}
\chi \equiv|\delta \omega|, & 0 \leqslant \chi \leqslant \infty, \\
\sigma \equiv|\sin \phi|, & 0 \leqslant \sigma \leqslant 1 .
\end{array}
$$

Then, with the further assumption that $\chi$ and $\sigma$ are statistically independent, we deduce that their joint probability density is

$$
\mathrm{q}(\chi, \sigma)=2\left(2 \pi\left\langle\delta \omega^{2}\right\rangle\right)^{1 / 2} \exp \left(-\frac{\chi^{2}}{2\left\langle\delta \omega^{2}\right\rangle}\right) \frac{2}{\pi}\left(1-\sigma^{2}\right)^{-1 / 2}
$$

The variable $\alpha$ is constant along hyperbolic contours in the $(\chi, \sigma)$ plane according to Equation (B.5). The total probability that $\alpha$ is less than a given value, $\alpha_{0}$, is, therefore

$$
\mathrm{p}\left\{\alpha<\alpha_{0}\right\}=\int_{0}^{1} \mathrm{~d} \sigma \int_{0}^{\alpha_{0} \sigma^{-1}\left(1-\mu_{\mathrm{R}}^{2}\right)^{-1 / 2}} \mathrm{~d} \dot{\chi} \mathrm{q}(\chi, \sigma)
$$

The probability density for $\alpha$ is

$$
\begin{aligned}
\psi\left(\alpha \mid \mu_{\mathrm{R}}\right) & =\left.\frac{\partial \mathrm{p}\left\{\alpha<\alpha_{\mathrm{o}}\right\}}{\partial \alpha_{\mathrm{o}}}\right|_{\alpha_{\mathrm{o}}=\alpha} ^{1} \\
& =\left(1-\mu_{\mathrm{R}}^{2}\right)^{-1 / 2} \int_{0}^{1} \mathrm{~d} \sigma \frac{1}{\sigma} \mathrm{q}\left[\frac{\alpha}{\sigma\left(1-\mu_{\mathrm{R}}^{2}\right)^{1 / 2}}, \sigma\right]
\end{aligned}
$$


with the function, q, given by Equation (B.6). Although the integration over $\sigma$ can be done analytically, reference to Equation (B. 3). shows :that only the first moment of $\psi\left(\alpha \mid \mu_{R}\right)$ is needed. Thus, when Equation (B. 8) with Equation (B.6) is substituted in Equation (B. 3) and the double integral evaluated, the result is

$$
\begin{gathered}
\mathrm{J}\left(\mu_{\mathrm{R}}\right)=\left\langle\alpha\left(\mu_{\mathrm{R}}\right)\right\rangle \mathrm{f}_{\mathrm{o}}\left(\mu_{\mathrm{R}}\right) \\
\left\langle\alpha\left(\mu_{\mathrm{R}}\right)\right\rangle=\left(\frac{2}{\pi}\right)^{3 / 2}\left\langle\delta \omega^{2}\right\rangle^{1 / 2}\left(1-\mu_{\mathrm{R}}^{2}\right)^{1 / 2} .
\end{gathered}
$$

In a system described by a diffusion equation like Equation.(30), conservation of particles requires that the particle flux be given by

$$
J(\mu)=-D_{\mu \mu} \frac{\partial f_{o}}{\partial \mu} .
$$

Combination of Equations (B.9) and (B.11) then gives the boundary condition on $f_{0}$ :

$$
\left.\left[\mathrm{D}_{\mu \mu} \frac{\partial \mathrm{f}_{\mathrm{O}}}{\partial \mu}+\langle\alpha\rangle \mathrm{f}_{\mathrm{o}}\right]\right|_{\mu=\mu_{\mathrm{R}}}=\mathrm{O}, \mu_{\mathrm{R}}>\mu_{\mathrm{S}} .
$$

Equation (B. 12) was derived for the case $\mu_{\mathrm{R}}>\mu_{\mathrm{S}}$. A similar derivation for $\mu_{\mathrm{L}}<\mu_{\mathrm{S}}$ results in the boundary condition

$$
\left.\left[\mathrm{D}_{\mu \mu} \frac{\partial \mathrm{f}_{\mathrm{O}}}{\partial \mu}-\langle\alpha\rangle \mathrm{f}_{\mathrm{o}}\right]\right|_{\mu=\mu_{\mathrm{L}}}=0, \mu_{\mathrm{L}}\left\langle\mu_{\mathrm{S}} .\right.
$$

In the presence of absorbing boundaries the Green's Function $\langle G\rangle_{R}$ also satisfies conditions (B.12) and (B.13). 
These boundary conditions have a simple physical interpretation. If the diffusion coefficient is used to define a "mean free path" for scattering in. $\mu-$ space as

$$
\begin{aligned}
\lambda & \equiv \mathrm{D}_{\mu \mu}\left\langle\frac{\mathrm{d} \mu}{\mathrm{dt}}\right\rangle^{-1}, \\
& =\mathrm{D}_{\mu \mu}\left\langle\alpha(\mu) \gamma^{-1},\right.
\end{aligned}
$$

then Equations (B.12), (B. 13) can be written

$$
\left.\left[\frac{\partial \mathrm{f}_{\mathrm{o}}}{\partial \mu} \pm \frac{1}{\lambda} \mathrm{f}_{\mathrm{o}}\right]\right|_{\mu=\mu_{\mathrm{R}}, \mu_{\mathrm{L}}}=0 .
$$

Equation (B.15) says that $f_{0}$ vanishes at a point one mean free path outside the absorbing boundary. In this form the boundary conditions closely resemble those given by Morse and Feshbach ${ }^{7}$ in an analysis of spatial diffusion near an absorbing boundary. 


\section{APPENDIX C}

\section{ERROR ANALYSIS}

The uncertainty in the value of the diffusion coefficient derived from .Equation (33) is a composite of the statistical uncertainties in $J$ and $d\langle f\rangle_{R} / d \mu$. The ensemble averaged flux is

$$
\langle\mathrm{J}\rangle=\frac{1}{\mathrm{R}} \sum_{\ell=1}^{\mathrm{R}} \mathrm{J}(\ell)
$$

where $J^{(\ell)}$ is the flux observed in the $\ell$-th realization. The uncertainty in $\langle J\rangle$ is taken to be

$$
\sigma_{\mathrm{J}}=\left\{\frac{1}{\mathrm{R}-1} \sum_{\ell=1}^{\mathrm{R}}\left[\mathrm{J}^{(\ell)}-\langle\mathrm{J}\rangle\right]^{2}\right\}^{1 / 2} .
$$

Similarly, if $n_{i}^{(\ell)}$ is the number of particles in the $i$-th histogram bin (width $\Delta \mu$, centered at $\mu_{\mathrm{i}}$ ) in the $\ell$-th realization, the ensemble averaged distribution function is

$$
\langle\mathrm{f}\rangle_{\mathrm{R}}\left(\mu_{\mathrm{i}}\right)=\frac{1}{\Delta \mu} \frac{\mathrm{i}}{\mathrm{R}} \sum_{\ell=1}^{\mathrm{R}} \mathrm{n}_{\mathrm{i}}^{(\mathrm{l})}
$$

with uncertainty

$$
\sigma_{f_{\mathrm{i}}}=\left\{\frac{1}{(\Delta \mu)^{2}} \frac{1}{\mathrm{R}-1} \sum_{\ell=1}^{\mathrm{R}}\left[\mathrm{n}_{\mathrm{i}}^{(\ell)}-\langle\mathrm{f}\rangle_{\mathrm{R}}\left(\mu_{\mathrm{i}}\right) \Delta \mu\right]^{2}\right\}^{3 / 2}
$$

Typically, $\sigma_{\mathrm{J}} /\langle\mathrm{J}\rangle$ and $\sigma_{\mathrm{f}_{\mathrm{i}}} /\langle\mathrm{f}\rangle_{\mathrm{R}}\left(\mu_{\mathrm{i}}\right)$ were a few percent or less.

The gradient of $\langle f\rangle_{\mathrm{R}}$ at $\mu, \mathrm{d}\langle\mathrm{f}\rangle_{\mathrm{R}} / \mathrm{d} \mu$, was taken to be the coefficient of the linear term in a weighted linear least-squares fit to the ten or so points, 
$\langle\mathrm{f}\rangle_{\mathrm{R}}\left(\mu_{\mathrm{i}}\right)$, symmetrically bracketing $\mu$. The uncertainty in $\mathrm{d}\langle\mathrm{f}\rangle_{\mathrm{R}} / \mathrm{d} \mu$ is then given by the standard formula ${ }^{9}$

$$
\sigma_{\mathrm{d}\langle\mathrm{f}\rangle_{\mathrm{R}} / \mathrm{d} \mu}^{2}=\frac{\sum_{\mathrm{i}} \frac{1}{\sigma_{\mathrm{f}_{i}}^{2}}}{\sum_{\mathrm{i}} \frac{1}{\sigma_{\mathrm{f}_{i}}^{2}} \sum_{\mathrm{i}} \frac{\mu_{i}^{2}}{\sigma_{f_{i}}^{2}}-\left[\sum_{i} \frac{\mu_{i}}{\sigma_{f_{i}}^{2}}\right]^{2}} .
$$

The resultant uncertainty in $\mathrm{D}_{\mu \mu}(\mu, \infty)$, then, is

$$
\frac{\sigma_{\mathrm{D}}}{\mathrm{D}_{\mu \mu}}=\left[\frac{\sigma_{\mathrm{J}}^{2}}{\langle\mathrm{~J}\rangle^{2}}+\frac{\sigma_{\mathrm{d}\langle\mathrm{f}\rangle_{\mathrm{R}} / \mathrm{d} \mu}^{2}}{\left(\mathrm{~d}\langle\mathrm{f}\rangle_{\mathrm{R}} / \mathrm{d} \mu\right)^{2}}\right]^{1 / 2}
$$

The number of points actually used in the fit was adjusted to give $\sigma_{\mathrm{D}} / \mathrm{D}_{\mu \mu} \cong .10$, provided that this did not require an unacceptably large spread in $\mu$. 